\title{
K- AND L-THEORY OF GRAPH PRODUCTS OF GROUPS
}

\author{
DANIEL KASPROWSKI, KEVIN LI, AND WOLFGANG LÜCK
}

\begin{abstract}
We compute the group homology, the algebraic $K$ - and $L$-groups, and the topological $K$-groups of right-angled Artin groups, right-angled Coxeter groups, and more generally, graph products.
\end{abstract}

\section{INTRODUCTION}

1.1. Basic setup. Suppose that we are given the following data:

- A finite simplicial graph $X$ on the vertex set $V$ and a collection of groups $\mathcal{W}:=\left\{W_{v} \mid v \in V\right\}$. Denote by $W=W(X, \mathcal{W})$ the associated graph product, see Section 3, and by $\Sigma$ the flag complex associated to $X$. Examples of graph products are right-angled Artin groups and right-angled Coxeter groups;

- A commutative ring with unit $\Lambda$ and an equivariant homology theory $\mathcal{H}_{*}^{\text {? }}$ with values in $\Lambda$-modules, see Definition 2.1. Our main examples will be those associated to algebraic $K$ - and $L$-theory or topological $K$-theory, which appear in the Farrell--Jones Conjecture or the Baum-Connes Conjecture;

- A non-empty class $\mathcal{C}$ of finite groups which is closed under isomorphisms, passage to subgroups and passage to quotient groups. Our main example will be the class of all finite groups;

- A class $\mathcal{E}$ of $\Lambda$-modules with the property that for an exact sequence $0 \rightarrow$ $V_{0} \rightarrow V_{1} \rightarrow V_{2} \rightarrow 0$ the $\Lambda$-module $V_{1}$ belongs to $\mathcal{E}$ if and only if both $V_{0}$ and $V_{2}$ belong to $\mathcal{E}$.

1.2. Main result. Fix an integer $n$. We obtain a covariant functor

$$
\mathcal{H}_{n}^{\mathcal{C}} \text { : Groups } \rightarrow \Lambda \text {-Modules, } \quad G \mapsto \mathcal{H}_{n}^{G}\left(E_{\mathcal{C}}(G)\right),
$$

where $E_{\mathcal{C}}(G)$ is the classifying space of the family of subgroups of $G$ which belong to $\mathcal{C}$, see Section 2 and (2.4).

Let $\mathcal{S}$ be the poset of flag subcomplexes of $\Sigma$ and let $\mathcal{P}$ be the poset of simplices of $\Sigma$, both ordered by inclusion, where the empty subcomplex and the empty simplex are allowed. For an element $L$ in $\mathcal{S}$, we can consider the subgraph $X \cap L$ of $X$. Let $W(L)$ be the graph product associated to $X \cap L$ and the collection of groups $\left.\mathcal{W}\right|_{V \cap L}=\left\{W_{v} \mid v\right.$ is a vertex of $\left.L\right\}$. With this notation $W(\Sigma)$ is the graph product $W(X, \mathcal{W})$ and $W(\emptyset)=\{1\}$. We obtain a covariant functor

$$
W_{*}: \mathcal{S} \rightarrow \text { Groups }, \quad L \mapsto W(L) .
$$

Let

$$
I: \mathcal{P} \rightarrow \mathcal{S}
$$

be the inclusion which sends a simplex $\sigma$ of $\Sigma$ to the corresponding flag subcomplex of $\Sigma$. Sometimes we identify $\sigma$ in $\mathcal{P}$ with $I(\sigma)$ in $\mathcal{S}$. For instance we will often write

Date: June 6, 2019.

2010 Mathematics Subject Classification. 18F25, 20F36, 20 F55.

Key words and phrases. $K$ - and $L$-groups, right-angled Artin and Coxeter groups. 
$W(\sigma)$ instead of $W(I(\sigma))$. Notice that the covariant functor $W_{*} \circ I: \mathcal{P} \rightarrow$ Groups sends a simplex $\sigma$ of $\Sigma$ to $\prod_{v \in V \cap \sigma} W_{v}$.

We obtain a covariant functor

$$
\mathcal{H}_{n}^{\mathcal{C}} \circ W_{*}: \mathcal{S} \rightarrow \Lambda \text {-Modules }, \quad L \mapsto \mathcal{H}_{n}^{W(L)}\left(E_{\mathcal{C}}(W(L))\right) .
$$

We are interested in the value at $\Sigma$, i.e., in $\mathcal{H}_{n}^{W}\left(E_{\mathcal{C}}(W)\right)$ for $W=W(X, \mathcal{W})$.

The composite $\mathcal{H}_{n}^{\mathcal{C}} \circ W_{*} \circ I: \mathcal{P} \rightarrow \Lambda$-Modules is given by

$$
\sigma \mapsto \mathcal{H}_{n}^{\mathcal{C}}\left(\prod_{v \in V \cap \sigma} W_{v}\right)=\mathcal{H}_{n}^{\prod_{v \in V \cap \sigma} W_{v}}\left(\prod_{v \in V \cap \sigma} E_{\mathcal{C}}\left(W_{v}\right)\right),
$$

since $\prod_{v \in V \cap \sigma} E_{\mathcal{C}}\left(W_{v}\right)$ is a model for $E_{\mathcal{C}}\left(\prod_{v \in V \cap \sigma} W_{v}\right)$.

Define for a simplex $\sigma$ the quotient $\Lambda$-module of $\mathcal{H}_{n}^{\mathcal{C}} \circ W_{*} \circ I(\sigma)$ by

$$
S_{\sigma}\left(\mathcal{H}_{n}^{\mathcal{C}} \circ W_{*} \circ I\right):=\operatorname{cok}\left(\bigoplus_{\tau} \mathcal{H}_{n}^{\mathcal{C}} \circ W_{*} \circ I(\tau) \rightarrow \mathcal{H}_{n}^{\mathcal{C}} \circ W_{*} \circ I(\sigma)\right),
$$

where $\tau$ runs through the simplices of $I(\sigma)$ which are different from $\sigma$. The idea is to kill everything in $\mathcal{H}_{n}^{\mathcal{C}} \circ W_{*} \circ I(\sigma)$ which comes from a proper simplex $\tau$ of $I(\sigma)$.

For a simplex $\sigma$ of $\Sigma$, let $\operatorname{ch}_{n}(\Sigma, \sigma)$ be the set of $n$-chains $\sigma_{0}<\sigma_{1}<\cdots<\sigma_{n}$ in $\mathcal{P}$ with $\sigma_{0}=\sigma$. Define the integer

$$
n_{\sigma}:=\sum_{n \geq 0}(-1)^{n} \cdot\left|\operatorname{ch}_{n}(\Sigma, \sigma)\right| .
$$

Denote by $G_{0}(\mathcal{E})$ the Grothendieck group of elements in $\mathcal{E}$, i.e., the abelian group with the isomorphism classes of elements in $\mathcal{E}$ as generators and relations $\left[V_{1}\right]=\left[V_{0}\right]+\left[V_{2}\right]$ for every short exact sequence $0 \rightarrow V_{0} \rightarrow V_{1} \rightarrow V_{2} \rightarrow 0$ of $\Lambda$-modules belonging to $\mathcal{E}$.

Theorem 1.1 (Main Theorem).

(i) The canoncial $\Lambda$-homomorphism

$$
T: \operatorname{colim}_{\sigma \in \mathcal{P}} \mathcal{H}_{n}^{W(\sigma)}\left(E_{\mathcal{C}}(W(\sigma))\right) \stackrel{\cong}{\rightrightarrows} \mathcal{H}_{n}^{W(\Sigma)}\left(E_{\mathcal{C}}(W(\Sigma))\right)
$$

is an isomorphism;

(ii) For every $\sigma \in \mathcal{P}$, the canonical projection

$$
p_{\sigma}: \mathcal{H}_{n}^{\mathcal{C}} \circ W_{*} \circ I(\sigma) \rightarrow S_{\sigma}\left(\mathcal{H}_{n}^{\mathcal{C}} \circ W_{*} \circ I\right)
$$

has a section

$$
s_{\sigma}: S_{\sigma}\left(\mathcal{H}_{n}^{\mathcal{C}} \circ W_{*} \circ I\right) \rightarrow \mathcal{H}_{n}^{\mathcal{C}} \circ W_{*} \circ I(\sigma) .
$$

Any collection of such sections and the canonical maps $\mathcal{H}_{n}^{\mathcal{C}} \circ W_{*} \circ I(\sigma) \rightarrow$ $\operatorname{colim}_{\sigma \in \mathcal{P}} \mathcal{H}_{n}^{\mathcal{C}} \circ W_{*} \circ I$ induce an isomorphism

$$
\bigoplus_{\sigma \in \mathcal{P}} S_{\sigma}\left(\mathcal{H}_{n}^{\mathcal{C}} \circ W_{*} \circ I\right) \stackrel{\cong}{\rightarrow} \operatorname{colim}_{\sigma \in \mathcal{P}} \mathcal{H}_{n}^{W(\sigma)}\left(E_{\mathcal{C}}(W(\sigma))\right) .
$$

Moreover, there is an explicit section $s_{\sigma}$;

(iii) Suppose that each $\Lambda$-module $\mathcal{H}_{n}^{W(\sigma)}\left(E_{\mathcal{C}}(W(\sigma))\right)$ belongs to $\mathcal{E}$. Then we get in $G_{0}(\mathcal{E})$

$$
\left[\mathcal{H}_{n}^{W(\Sigma)}\left(E_{\mathcal{C}}(W(\Sigma))\right)\right]=\sum_{\sigma \in \mathcal{P}} n_{\sigma} \cdot\left[\mathcal{H}_{n}^{W(\sigma)}\left(E_{\mathcal{C}}(W(\sigma))\right)\right] .
$$

We mention that it is both unusual and fortunate that in assertion (i) the source and target involve the same degree. In general one would expect that in the source all degrees $m \leq n$ occur. The reason for this simplification is that for each simplex $\sigma$ the inclusion $W(\sigma) \rightarrow W$ is split injective. This leads also to the explicit splitting in assertion (ii). 
Note that the number $n_{\sigma}$ appearing in assertion (iii) depends only on $\Sigma$. It is given by $1-\chi(\Sigma)$ for $\sigma=\emptyset$. It has the following geometric interpretation if $\sigma$ is non-empty. Let $\Sigma^{\prime}$ be the barycentric subdivision of $\Sigma$. Then $\operatorname{ch}_{n}(\Sigma, \sigma)$ can be interpreted as a collection of $n$-simplices in $\Sigma^{\prime}$. Each simplex in $\operatorname{ch}_{n}(\Sigma, \sigma)$ contains the vertex given by $\sigma$. The collection of the faces of all these $\operatorname{simplices}$ of $\operatorname{ch}_{n}(\Sigma, \sigma)$ determines a simplicial subcomplex $D_{\sigma}$ of $\Sigma^{\prime}$ which can be contracted to the vertex given by $\sigma$. Its boundary $\partial D_{\sigma}$ consists of all those faces of $\operatorname{simplices}$ of $\operatorname{ch}_{n}(\Sigma, \sigma)$ which do not contain $\sigma$. One easily checks

$$
n_{\sigma}=\chi\left(D_{\sigma}\right)-\chi\left(\partial D_{\sigma}\right)=1-\chi\left(\partial D_{\sigma}\right)
$$

If $\Sigma$ is the triangulation of a closed manifold of dimension $d$, then $\partial D_{\sigma}$ is homeomorphic to $S^{d-1-\operatorname{dim}(\sigma)}$ and hence $n_{\sigma}=(-1)^{d-\operatorname{dim}(\sigma)}$.

1.3. Computations. We will illustrate the potential of Theorem 1.1 by computing the group homology of $G$, the algebraic $K$ - and $L$-theory of the group $\operatorname{ring}$ of $G$, and the topological $K$-theory of the group $C^{*}$-algebra of $G$ in Sections 6 and 7 if $G$ is a right-angled Artin group or a right-angled Coxeter group. These computations are based on the Baum-Connes Conjecture and the Farrell--Jones Conjecture which we will briefly recall in Section 5 and which hold for these groups. The situation in the Farrell-Jones setting is more complicated since we have to deal with the family $\mathcal{V C Y}$ of virtually cyclic subgroups, whereas in Theorem 1.1 the family $\mathcal{F} \mathcal{I} \mathcal{N}$ of finite subgroups is considered. The passage from $\mathcal{F} \mathcal{I N}$ to $\mathcal{V} \mathcal{Y}$ is discussed in Subsection 5.3. This is different in the Baum-Connes setting since there the family $\mathcal{F} \mathcal{I} \mathcal{N}$ is used. In order to get full functoriality we need to consider the maximal group $C^{*}$-algebra instead of the reduced $C^{*}$-algebra which makes no difference for right-angled Artin groups and right-angled Coxeter groups.

Computation in this context means not only that we identify the corresponding $K$ - and $L$-groups of $G$ as abelian groups but we give explicit isomorphisms identifying them with $K$ - and $L$-groups of the ground ring. For instance, we show for a right-angled Coxeter group $W$ associated to the finite flag complex $\Sigma$ that there is for every $n \in \mathbb{Z}$ an isomorphism

$$
\bigoplus_{\sigma} K_{n}\left(f_{\sigma}\right): \bigoplus_{\sigma} K_{n}(\mathbb{C}) \stackrel{\cong}{\longrightarrow} K_{n}\left(C_{r}^{*}(W)\right)
$$

where $\sigma$ runs through the simplices of $\Sigma$ including the empty simplex and $f_{\sigma}: \mathbb{C} \rightarrow$ $C_{r}^{*}(W)$ is an explicit homomorphism of $C^{*}$-algebras depending on $\sigma$. If $\sigma$ is empty, it is given by the obvious inclusion $\mathbb{C} \rightarrow C_{r}^{*}(W)$. If $k=\operatorname{dim}(\sigma) \geq 0$, then $\sigma$ determines a subgroup $W(\sigma)=\prod_{i=1}^{k+1} \mathbb{Z} / 2$ of $W$ and $f_{\sigma}$ is the composite of the homomorphism $\mathbb{C} \rightarrow \mathbb{C}\left[\prod_{i=1}^{k+1} \mathbb{Z} / 2\right]$ sending $\lambda$ to $2^{-k-1} \cdot \lambda \cdot \prod_{i=1}^{k+1}\left(1-t_{i}\right)$ for $t_{i}$ the generator of the $i$-th factor $\mathbb{Z} / 2$ and the homomorphism $\mathbb{C}[W(\sigma)] \rightarrow C_{r}^{*}(W)$ coming from the inclusion $W(\sigma) \rightarrow W$, see Remark 7.17. This implies

$$
K_{n}\left(C_{r}^{*}(W)\right) \cong \begin{cases}\mathbb{Z}^{r} & \text { if } n \text { is even } \\ \{0\} & \text { otherwise }\end{cases}
$$

where $r$ is the number of simplices of $\Sigma$ including the empty simplex. Moreover, we can write down an explicit basis $B=\left\{b_{\sigma} \mid \sigma \in \mathcal{P}\right\}$ for the finitely generated free $\mathbb{Z}$-module $K_{0}\left(C_{r}^{*}(W)\right.$ ), namely, for $\sigma=\emptyset$ we take the class of the idempotent 1 in $C_{r}^{*}(W)$ and for $\sigma \neq \emptyset$ we take the class of the idempotent in $C_{r}^{*}(W)$ given by the image of the idempotent $2^{-k-1} \cdot \prod_{i=1}^{k+1}\left(1-t_{i}\right) \in \mathbb{C}[W(\sigma)]$ under the inclusion $\mathbb{C}[W(\sigma)] \subset C_{r}^{*}(W)$. These computations for right-angled Coxeter groups were carried out in the second author's master's thesis [22]. 
1.4. Acknowledgments. The paper was financially supported by the ERC Advanced Grant "KL2MG-interactions" (no. 662400) of the third author granted by the European Research Council. It was also funded by the Deutsche Forschungsgemeinschaft (DFG, German Research Foundation) under Germany's Excellence Strategy - GZ 2047/1, Projekt-ID 390685813.

\section{Contents}

1. Introduction 1

1.1. Basic setup 1

1.2. Main result 1

1.3. Computations 3

1.4. Acknowledgments 4

2. Equivariant homology theories and classifying spaces of families 4

3. Graph products of groups $\quad 7$

4. Mackey modules 10

4.1. Proof of Theorem 1.1 14

5. Isomorphism Conjectures in $K$ - and $L$-theory 14

5.1. The Baum-Connes Conjecture 15

5.2. The Farrell-Jones Conjecture 15

5.3. The passage from $\mathcal{F} \mathcal{I N}$ to $\mathcal{V C Y} \quad 16$

6. Right-angled Artin groups 18

6.1. Group homology 19

6.2. Algebraic $K$-theory 20

6.3. Algebraic $L$-theory 20

6.4. Topological $K$-theory 20

7. Right-angled Coxeter groups 20

7.1. Group homology 21

7.2. Negative $K$-groups for $R=\mathbb{Z} \quad 23$

7.3. Projective class group for $R=\mathbb{Z} \quad 23$

$\begin{array}{ll}\text { 7.4. Whitehead group } & 24\end{array}$

7.5. Rationalized $K$-groups 24

7.6. $L$-groups after inverting $2 \quad 26$

7.7. $K$ - and $L$-groups for $R$ containing $1 / 2 \quad 26$

7.8. Topological $K$-theory 29

8. An example 29

References $\quad 31$

\section{Equivariant HOMOLOGY THEORIES AND CLASSIFYing SPACES OF FAMILIES}

In this section we recall the axioms of an equivariant homology theory and the notion of a classifying space of a family of subgroups. For an amalgamated product of groups, we deduce a Mayer-Vietoris type sequence for the values of an equivariant homology theory on classifying spaces.

Fix a discrete group $G$ and a commutative ring $\Lambda$ with unit. A G-homology theory $\mathcal{H}_{*}^{G}$ with values in $\Lambda$-modules is a collection of covariant functors $\mathcal{H}_{n}^{G}$ from the category of $G-C W$-pairs to the category of $\Lambda$-modules indexed by $n \in \mathbb{Z}$ together with natural transformations

$$
\partial_{n}^{G}(X, A): \mathcal{H}_{n}^{G}(X, A) \rightarrow \mathcal{H}_{n-1}^{G}(A):=\mathcal{H}_{n-1}^{G}(A, \emptyset)
$$

for $n \in \mathbb{Z}$ such that the axioms concerning $G$-homotopy invariance, the long exact sequence of a pair, excision, and disjoint unions are satisfied, see [24, Section 1]. 
Let $\alpha: H \rightarrow G$ be a group homomorphism. Given an $H$-space $X$, define the induction of $X$ with $\alpha$ to be the $G$-space $\alpha_{*} X=G \times{ }_{\alpha} X$ which is the quotient of $G \times X$ by the right $H$-action $(g, x) \cdot h:=\left(g \alpha(h), h^{-1} x\right)$ for $h \in H$ and $(g, x) \in G \times X$. The following definition is taken from [24, Section 1] except that the induction structure in this paper is defined for every group homomorphism $\alpha$.

Definition 2.1. An equivariant homology theory $\mathcal{H}_{*}^{\text {? }}$ with values in $\Lambda$-modules assigns to each group $G$ a $G$-homology theory $\mathcal{H}_{*}^{G}$ with values in $\Lambda$-modules together with the following so called induction structure:

Given a group homomorphism $\alpha: H \rightarrow G$ and an $H$-CW-pair $(X, A)$, there are for every $n \in \mathbb{Z}$ natural homomorphisms

$$
\operatorname{ind}_{\alpha}: \mathcal{H}_{n}^{H}(X, A) \rightarrow \mathcal{H}_{n}^{G}\left(\alpha_{*}(X, A)\right)
$$

satisfying:

- Compatibility with the boundary homomorphisms:

$\partial_{n}^{G} \circ \operatorname{ind}_{\alpha}=\operatorname{ind}_{\alpha} \circ \partial_{n}^{H}$

- Functoriality:

Let $\beta: G \rightarrow K$ be another group homomorphism. Then we have for $n \in \mathbb{Z}$ $\operatorname{ind}_{\beta \circ \alpha}=\mathcal{H}_{n}^{K}\left(f_{1}\right) \circ \operatorname{ind}_{\beta} \circ \operatorname{ind}_{\alpha}: \mathcal{H}_{n}^{H}(X, A) \rightarrow \mathcal{H}_{n}^{K}\left((\beta \circ \alpha)_{*}(X, A)\right)$,

where $f_{1}: \beta_{*}\left(\alpha_{*}(X, A)\right) \stackrel{\cong}{\longrightarrow}(\beta \circ \alpha)_{*}(X, A), \quad(k, g, x) \mapsto(k \beta(g), x)$ is the natural $K$-homeomorphism;

- Compatibility with conjugation:

For $n \in \mathbb{Z}, g \in G$ and a $G$-CW-pair $(X, A)$ the map

$$
\operatorname{ind}_{c(g): G \rightarrow G}: \mathcal{H}_{n}^{G}(X, A) \rightarrow \mathcal{H}_{n}^{G}\left(c(g)_{*}(X, A)\right)
$$

agrees with $\mathcal{H}_{n}^{G}\left(f_{2}\right)$ for the $G$-homeomorphism $f_{2}:(X, A) \rightarrow c(g)_{*}(X, A)$ which sends $x$ to $\left(1, g^{-1} x\right)$ in $G \times_{c(g)}(X, A)$;

- Bijectivity:

If $\operatorname{ker}(\alpha)$ acts freely on $X \backslash A$, then $\operatorname{ind}_{\alpha}: \mathcal{H}_{n}^{H}(X, A) \rightarrow \mathcal{H}_{n}^{G}\left(\alpha_{*}(X, A)\right)$ is bijective for all $n \in \mathbb{Z}$.

We briefly fix some conventions concerning spectra. If $X$ is a space, denote by $X_{+}$the pointed space obtained from $X$ by adding a disjoint base point. Let Spectra be the category of spectra in the following naive sense. A spectrum $\mathbf{E}=$ $\{(E(n), \sigma(n)) \mid n \in \mathbb{Z}\}$ is a sequence of pointed spaces $\{E(n) \mid n \in \mathbb{Z}\}$ together with pointed maps called structure maps $\sigma(n): E(n) \wedge S^{1} \longrightarrow E(n+1)$. A map of spectra $\mathbf{f}: \mathbf{E} \rightarrow \mathbf{E}^{\prime}$ is a sequence of maps $f(n): E(n) \rightarrow E^{\prime}(n)$ which are compatible with the structure maps $\sigma(n)$, i.e., we have $f(n+1) \circ \sigma(n)=\sigma^{\prime}(n) \circ\left(f(n) \wedge \mathrm{id}_{S^{1}}\right)$ for all $n \in \mathbb{Z}$. Given a spectrum $\mathbf{E}$ and a pointed space $X$, we can define their smash product $X \wedge \mathbf{E}$ by $(X \wedge \mathbf{E})(n):=X \wedge E(n)$ with the obvious structure maps.

It is a classical result that a spectrum $\mathbf{E}$ defines a homology theory by setting

$$
H_{n}(X, A ; \mathbf{E})=\pi_{n}\left(\left(X_{+} \cup_{A_{+}} \operatorname{cone}\left(A_{+}\right)\right) \wedge \mathbf{E}\right),
$$

where cone denotes the reduced cone. We want to extend this to equivariant homology theories.

Let Groupoids be the category of small connected groupoids with covariant functors as morphisms. Notice that a group can be considered as a groupoid with one object in the obvious way.

For the proof of the following result we refer to [27, Proposition 157 on page 796].

Theorem 2.2. Consider a covariant Groupoids-spectrum

$$
\text { E: Groupoids } \rightarrow \text { Spectra. }
$$


Suppose that $\mathbf{E}$ respects equivalences, i.e., it sends an equivalence of groupoids to a weak equivalence of spectra.

Then $\mathbf{E}$ defines an equivariant homology theory $H_{*}^{?}(-; \mathbf{E})$ such that we have

$$
H_{n}^{G}(G / H ; \mathbf{E}) \cong H_{n}^{H}(\mathrm{pt} ; \mathbf{E}) \cong \pi_{n}(\mathbf{E}(H))
$$

for every group $G$, subgroup $H \subseteq G$ and $n \in \mathbb{Z}$. The construction is natural in $\mathbf{E}$.

Example 2.3 (Borel homology). Let $\mathbf{E}$ be a spectrum. Let $H_{*}(-; \mathbf{E})$ be the (nonequivariant) homology theory associated to $\mathbf{E}$. Given a groupoid $\mathcal{G}$, denote by $E \mathcal{G}$ its classifying space. If $\mathcal{G}$ has only one object and the automorphism group of this object is $G$, then $E \mathcal{G}$ is a model for $E G$. We obtain two covariant functors

$$
\begin{array}{ll}
c_{\mathbf{E}}: \text { Groupoids } \rightarrow \text { Spectra }, & \mathcal{G} \mapsto \mathbf{E} ; \\
b_{\mathbf{E}}: \text { Groupoids } \rightarrow \text { Spectra }, & \mathcal{G} \mapsto E \mathcal{G}_{+} \wedge \mathbf{E} .
\end{array}
$$

Thus we obtain two equivariant homology theories $H_{*}^{?}\left(-; c_{\mathbf{E}}\right)$ and $H_{*}^{?}\left(-; b_{\mathbf{E}}\right)$ from Theorem 2.2. The second one is called the equivariant Borel homology associated to $H_{*}(-; \mathbf{E})$. We get for any group $G$ and any $G-C W$-complex $X$ natural isomorphisms

$$
\begin{aligned}
& H_{n}^{G}\left(X ; c_{\mathbf{E}}\right) \cong H_{n}(G \backslash X ; \mathbf{E}) ; \\
& H_{n}^{G}\left(X ; b_{\mathbf{E}}\right) \cong H_{n}\left(E G \times_{G} X ; \mathbf{E}\right) .
\end{aligned}
$$

Let $\mathcal{C}$ be a non-empty class of groups which is closed under isomorphisms, passage to subgroups and passage to quotient groups. Our main examples will be the class $\mathcal{F} \mathcal{I}$ of finite groups and the class $\mathcal{V C Y}$ of virtually cyclic groups.

Given a group $G$, denote by $E_{\mathcal{C}}(G)$ the classifying space of $G$ with respect to the family of subgroups $\mathcal{F}_{\mathcal{C}}(G)=\{H \subseteq G \mid H \in \mathcal{C}\}$. It is defined to be a terminal object in the $G$-homotopy category of $G$-CW-complexes, whose isotropy groups belong to $\mathcal{F}_{\mathcal{C}}(G)$. A model for $E_{\mathcal{C}}(G)$ is a $G-C W$-complex whose $H$-fixed point set is contractible if $H \in \mathcal{C}$ and is empty if $H \notin \mathcal{C}$. With this notation $E_{\mathcal{F} \mathcal{I N}}(G)$ is the classifying space of proper actions, sometimes also denoted by $\underline{E} G$. We sometimes denote $E_{\mathcal{V} \mathcal{Y}}(G)$ by $\underline{\underline{E}} G$. For more information about classifying spaces of families we refer for instance to [26].

Given a group homomorphism $f: G \rightarrow H$, we denote by

$$
E_{\mathcal{C}}(f): f_{*} E_{\mathcal{C}}(G) \rightarrow E_{\mathcal{C}}(H)
$$

the up to $H$-homotopy unique $H$-map coming from the universal property of $E_{\mathcal{C}}(H)$ and the fact that $f_{*} E_{\mathcal{C}}(G)$ is an $H$-CW-complex whose isotropy groups are of the shape $f(K)$ for $K \in \mathcal{C}$ and hence all belong to $\mathcal{C}$ again. Given an equivariant homology theory $\mathcal{H}_{*}^{?}$ with values in $\Lambda$-modules, it induces homomorphisms of $\mathbb{Z}$ graded $\Lambda$-modules

$$
f_{*}: \mathcal{H}_{*}^{G}\left(E_{\mathcal{C}}(G)\right) \stackrel{\operatorname{ind}_{f}}{\longrightarrow} \mathcal{H}_{*}^{H}\left(f_{*} E_{\mathcal{C}}(G)\right) \stackrel{\mathcal{H}_{*}^{H}\left(E_{\mathcal{C}}(f)\right)}{\longrightarrow} \mathcal{H}_{*}^{H}\left(E_{\mathcal{C}}(H)\right) .
$$

One easily checks that thus we obtain a covariant functor

$$
\mathcal{H}_{n}^{\mathcal{C}}: \text { Groups } \rightarrow \Lambda \text {-Modules }, \quad G \mapsto \mathcal{H}_{n}^{G}\left(E_{\mathcal{C}}(G)\right) .
$$

Lemma 2.5. Let $G_{0}, G_{1}$ and $G_{2}$ be subgroups of $G$ satisfying $G_{0} \subseteq G_{1}, G_{2}$. Suppose that the inclusions $i_{k}: G_{k} \rightarrow G$ for $k=0,1,2$ induce an isomorphism $G_{1} * G_{0} G_{2} \cong G$. Let $j_{k}: G_{0} \rightarrow G_{k}$ be the inclusion for $k=1,2$. Suppose that each element in $\mathcal{C}$ is a finite group.

Then we obtain a long exact Mayer-Vietoris sequence

$$
\begin{aligned}
\cdots \stackrel{\partial_{n+1}}{\longrightarrow} \mathcal{H}_{n}^{G_{0}}\left(E_{\mathcal{C}}\left(G_{0}\right)\right) & \stackrel{\left(j_{1}\right)_{n} \times-\left(j_{2}\right)_{n}}{\longrightarrow} \mathcal{H}_{n}^{G_{1}}\left(E_{\mathcal{C}}\left(G_{1}\right)\right) \oplus \mathcal{H}_{n}^{G_{2}}\left(E_{\mathcal{C}}\left(G_{2}\right)\right) \\
\stackrel{\left(i_{1}\right)_{n} \oplus\left(i_{2}\right)_{n}}{\longrightarrow} & \mathcal{H}_{n}^{G}\left(E_{\mathcal{C}}(G)\right) \stackrel{\partial_{n}}{\longrightarrow} \mathcal{H}_{n-1}^{G_{0}}\left(E_{\mathcal{C}}\left(G_{0}\right)\right) \stackrel{\left(j_{1}\right)_{n-1} \times-\left(j_{2}\right)_{n-1}}{\longrightarrow} \cdots .
\end{aligned}
$$


Proof. There is a 1-dimensional $G$ - $C W$-complex $T$ whose underlying space is a tree such that the 1-skeleton is obtained from the 0 -skeleton by the $G$-pushout

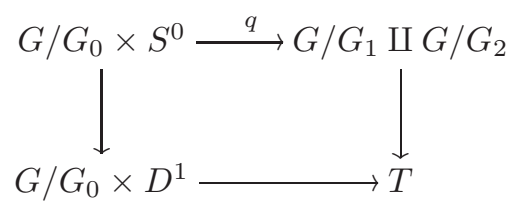

where $q$ is the disjoint union of the canonical projections $G / G_{0} \rightarrow G / G_{1}$ and $G / G_{0} \rightarrow G / G_{2}$, see [35, Theorem 7 in $\S 4.1$ on page 32]. If we take the cartesian product with $E_{\mathcal{C}}(G)$ we obtain another cellular $G$-pushout. Its associated MayerVietoris sequence yields the long exact sequence

$$
\begin{aligned}
\cdots \rightarrow \mathcal{H}_{n}^{G}\left(G / G_{0} \times E_{\mathcal{C}}(G)\right) & \rightarrow \mathcal{H}_{n}^{G}\left(G / G_{1} \times E_{\mathcal{C}}(G)\right) \oplus \mathcal{H}_{n}^{G}\left(G / G_{2} \times E_{\mathcal{C}}(G)\right) \\
\rightarrow & \mathcal{H}_{n}^{G}\left(T \times E_{\mathcal{C}}(G)\right) \rightarrow \mathcal{H}_{n-1}^{G}\left(G / G_{0} \times E_{\mathcal{C}}(G)\right) \rightarrow \cdots .
\end{aligned}
$$

There is a $G$-homeomorphism $\left(j_{k}\right)_{*} j_{k}^{*} E_{\mathcal{C}}(G) \stackrel{\cong}{\rightrightarrows} G / G_{k} \times E_{\mathcal{C}}(G)$, where $j_{k}^{*} E_{\mathcal{C}}(G)$ is the restriction of $E_{\mathcal{C}}(G)$ to $G_{k}$ by $j_{k}$. Obviously $j_{k}^{*} E_{\mathcal{C}}(G)$ is a model for $E_{\mathcal{C}}\left(G_{k}\right)$. Using the induction structure of the equivariant homology theory $\mathcal{H}_{*}^{\text {? }}$ we obtain identifications for $k=0,1,2$

$$
\mathcal{H}_{*}^{G_{k}}\left(E_{\mathcal{C}}\left(G_{k}\right)\right) \stackrel{\cong}{\longrightarrow} \mathcal{H}_{*}^{G}\left(G / G_{k} \times E_{\mathcal{C}}(G)\right) .
$$

The $K$-fixed point set $T^{K}$ is a non-empty subtree and hence contractible for every finite subgroup $K \subseteq G$, see [35, Theorem 15 in 6.1 on page 58 and 6.3 .1 on page 60 ]. Hence the projection $T \times E_{\mathcal{C}}(G) \rightarrow E_{\mathcal{C}}(G)$ is a $G$-homotopy equivalence since every element in $\mathcal{C}$ is finite by assumption. Hence we get an identification

$$
\mathcal{H}_{*}^{G}\left(E_{\mathcal{C}}(G)\right) \stackrel{\cong}{\longrightarrow} \mathcal{H}_{*}^{G}\left(T \times E_{\mathcal{C}}(G)\right) .
$$

Now we obtain the desired long exact sequence from the last long exact sequence and the identifications above.

\section{Graph products of Groups}

In this section we give the definition of a graph product of groups. We show that the value of an equivariant homology theory on the classifying space of a graph product is the colimit over a certain system of subgroups.

Let $X$ be a finite simplicial graph on the vertex set $V$ and suppose that we are given a collection of groups $\mathcal{W}:=\left\{W_{v} \mid v \in V\right\}$. Then the graph product $W(X, \mathcal{W})$ is defined as the quotient of the free product $*_{v \in V} W_{v}$ of the collection of groups $\mathcal{W}$ by introducing the relations

$\left\{\left[g, g^{\prime}\right]=1 \mid v, v^{\prime} \in V\right.$, there is an edge joining $v$ and $\left.v^{\prime}, g \in W_{v}, g^{\prime} \in W_{v^{\prime}}\right\}$.

In other words, elements of subgroups $W_{v}$ and $W_{v^{\prime}}$ commute if there is an edge joining $v$ and $v^{\prime}$. This notion is due to Green [18].

Let $\Sigma$ be the flag complex associated to $X$. Denote by $\mathcal{S}=\mathcal{S}(\Sigma)$ the poset of flag subcomplexes of $\Sigma$, ordered by inclusion, where we also allow the empty subcomplex. Then we can assign to $L \in \mathcal{S}$ the graph product group $W(X \cap$ $\left.L,\left.\mathcal{W}\right|_{V \cap L}\right)$, where $X \cap L$ agrees with the 1-skeleton of $L$ and $\left.\mathcal{W}\right|_{V \cap L}=\left\{W_{v} \mid v \in\right.$ $V \cap L\}$ is the restriction of $\mathcal{W}$ to the vertices in $L$. Consider $L_{0}, L_{1} \in \mathcal{S}$ with $L_{0} \leq L_{1}$. Then we obtain group homomorphisms

$$
\begin{aligned}
W_{*}\left(L_{0} \leq L_{1}\right): W\left(L_{0},\left.\mathcal{W}\right|_{V \cap L_{0}}\right) & \rightarrow W\left(L_{1},\left.\mathcal{W}\right|_{V \cap L_{1}}\right) ; \\
W^{*}\left(L_{0} \leq L_{1}\right): W\left(L_{1},\left.\mathcal{W}\right|_{V \cap L_{1}}\right) & \rightarrow W\left(L_{0},\left.\mathcal{W}\right|_{V \cap L_{0}}\right)
\end{aligned}
$$


as follows. The morphism $W_{*}\left(L_{0} \leq L_{1}\right)$ is induced by the obvious inclusion $*_{v \in V \cap L_{0}} W_{v} \rightarrow *_{v \in V \cap L_{1}} W_{v}$, whereas the second one is induced by the projection $*_{v \in V \cap L_{1}} W_{v} \rightarrow *_{v \in V \cap L_{0}} W_{v}$ which is given on $W_{v}$ for $v \in V \cap L_{1}$ by the inclusion $W_{v} \rightarrow *_{v \in V \cap L_{0}} W_{v}$ if $v \in L_{0}$, and by the trivial homomorphism if $v \notin L_{0}$. One easily checks that thus we obtain a covariant functor

$$
W_{*}: \mathcal{S} \rightarrow \text { Groups }
$$

and a contravariant functor

$$
W^{*}: \mathcal{S} \rightarrow \text { Groups. }
$$

By construction $W_{*}$ and $W^{*}$ agree on objects and we write

$$
W(L):=W_{*}(L)=W^{*}(L):=W\left(X \cap L,\left.\mathcal{W}\right|_{V \cap L}\right)
$$

for an object $L \in \mathcal{S}$.

The elementary proof of the following lemma is left to the reader.

\section{Lemma 3.1.}

(i) Let $L_{0}, L_{1}, L_{2}, L \in \mathcal{S}$ be elements such that $L=L_{1} \cup L_{2}$ and $L_{0}=L_{1} \cap L_{2}$. Then we obtain a group isomorphism

$$
W_{*}\left(L_{1} \leq L\right) *_{W_{*}\left(L_{0} \leq L\right)} W_{*}\left(L_{2} \leq L\right): W\left(L_{1}\right) * W\left(L_{0}\right) W\left(L_{2}\right) \stackrel{\cong}{\longrightarrow} W(L) ;
$$

(ii) Let $L_{1}, L_{2}, L \in \mathcal{S}$ be elements satisfying $L_{1} \leq L$ and $L_{2} \leq L$. Then we get an equality of group homomorphisms $W\left(L_{1}\right) \rightarrow W\left(L_{2}\right)$

$$
W^{*}\left(L_{2} \leq L\right) \circ W_{*}\left(L_{1} \leq L\right)=W_{*}\left(\left(L_{1} \cap L_{2}\right) \leq L_{2}\right) \circ W^{*}\left(\left(L_{1} \cap L_{2}\right) \leq L_{1}\right) .
$$

Remark 3.2. Notice that in particular we get from Lemma 3.1 (ii) that for any two elements $L_{0}$ and $L_{1}$ in $\mathcal{S}$ with $L_{0} \leq L_{1}$ the composite $W^{*}\left(L_{0} \leq L_{1}\right) \circ W_{*}\left(L_{0} \leq L_{1}\right)$ is the identity on $W\left(L_{0}\right)$ and hence $W_{*}\left(L_{0} \leq L_{1}\right)$ is split injective and $W^{*}\left(L_{0} \leq L_{1}\right)$ is split surjective.

Let $\mathcal{H}_{*}^{?}$ be an equivariant homology theory with values in $\Lambda$-modules. Let $\mathcal{C}$ be a non-empty class of groups which is closed under isomorphisms, passage to subgroups and passage to quotient groups. We have defined a covariant functor $\mathcal{H}_{*}^{\mathcal{C}}$ and studied its main properties in Section 2.

We want to study the covariant $\Lambda \mathcal{S}$-module

$$
\mathcal{H}_{n}^{\mathcal{C}} \circ W_{*}: \mathcal{S} \rightarrow \Lambda \text {-Modules }, \quad L \mapsto \mathcal{H}_{n}^{W(L)}\left(E_{\mathcal{C}}(W(L))\right)
$$

and are in particular interested in its value at $\Sigma$ itself.

Viewing a simplex as a flag subcomplex yields for every $L \in \mathcal{S}$ a map of posets

$$
I_{L}: \mathcal{P}(L) \rightarrow \mathcal{S}(L) \text {. }
$$

For two elements $L$ and $L^{\prime}$ in $\mathcal{S}$ with $L \leq L^{\prime}$ let

$$
\begin{aligned}
& J^{\mathcal{P}}\left(L \leq L^{\prime}\right): \mathcal{P}(L) \quad \rightarrow \quad \mathcal{P}\left(L^{\prime}\right) ; \\
& J^{\mathcal{S}}\left(L \leq L^{\prime}\right): \mathcal{S}(L) \quad \rightarrow \quad \mathcal{S}\left(L^{\prime}\right)
\end{aligned}
$$

be the maps of posets induced by the inclusion $L \subseteq L^{\prime}$. Define the $\Lambda$-module

$$
C_{n}(L):=\operatorname{colim}_{\mathcal{P}(L)} \mathcal{H}_{n}^{\mathcal{C}} \circ W_{*} \circ J^{\mathcal{S}}(L \leq \Sigma) \circ I_{L}
$$

to be the colimit of the covariant functor $\mathcal{H}_{n}^{\mathcal{C}} \circ W_{*} \circ J^{\mathcal{S}}(L \leq \Sigma) \circ I_{L}: \mathcal{P}(L) \rightarrow$ $\Lambda$-Modules. Given elements $L$ and $L^{\prime}$ in $\mathcal{S}$ with $L \leq L^{\prime}$, we obtain a map of $\Lambda$-modules

$$
C_{n}\left(L \leq L^{\prime}\right): C_{n}(L) \rightarrow C_{n}\left(L^{\prime}\right)
$$

from $J^{\mathcal{P}}\left(L \leq L^{\prime}\right)$ because of $J^{\mathcal{S}}\left(L^{\prime} \leq \Sigma\right) \circ I_{L^{\prime}} \circ J^{\mathcal{P}}\left(L \leq L^{\prime}\right)=J^{\mathcal{S}}(L \leq \Sigma) \circ I_{L}$. One easily checks that thus we obtain a covariant $\Lambda \mathcal{S}$-module

$$
C_{n}: \mathcal{S} \rightarrow \Lambda \text {-Modules, } \quad L \mapsto C_{n}(L) .
$$


For every object $L \in \mathcal{S}$ there is an obvious $\Lambda$-homomorphism

$$
T_{n}(L): C_{n}(L)=\operatorname{colim}_{\mathcal{P}(L)} \mathcal{H}_{n}^{\mathcal{C}} \circ W_{*} \circ J^{\mathcal{S}}(L \leq \Sigma) \circ I_{L} \rightarrow \mathcal{H}_{n}^{\mathcal{C}} \circ W_{*}(L)
$$

coming from the various $\Lambda$-maps $\mathcal{H}_{n}^{\mathcal{C}} \circ W_{*}(\sigma) \rightarrow \mathcal{H}_{n}^{\mathcal{C}} \circ W_{*}(L)$ induced by the inclusions $I_{L}(\sigma) \subseteq L$ for $\sigma$ running through the simplices of $L$. One easily checks that the collection of the $\Lambda$-homomorphisms $T_{n}(L)$ fits together to a map of covariant $\Lambda \mathcal{S}$ modules

$$
T_{n}: C_{n} \rightarrow \mathcal{H}_{n}^{\mathcal{C}} \circ W_{*} .
$$

Theorem 3.4. Suppose that each element in $\mathcal{C}$ is a finite group.

Then the map of $\Lambda \mathcal{S}$-modules $T_{n}$ of (3.3) is an isomorphism. In particular its evaluation at $\Sigma$ yields a $\Lambda$-isomorphism

$$
T_{n}(\Sigma): \operatorname{colim}_{\sigma \in \mathcal{P}} \mathcal{H}_{n}^{W(\sigma)}\left(E_{\mathcal{C}}(W(\sigma))\right) \stackrel{\cong}{\rightrightarrows} \mathcal{H}_{n}^{W(\Sigma)}\left(E_{\mathcal{C}}(W(\Sigma))\right) .
$$

Proof. Notice that $T_{n}(L)$ is obviously an isomorphism if $L$ lies in the image of $I: \mathcal{P} \rightarrow \mathcal{S}$, since then $L$ is a terminal object in $\mathcal{P}(L)$ and hence under the obvious identification $C_{n}(L)=\operatorname{colim}_{\mathcal{P}(L)} \mathcal{H}_{n}^{\mathcal{C}} \circ W_{*} \circ J^{\mathcal{S}}(L \leq \Sigma) \circ I_{L} \cong \mathcal{H}_{n}^{\mathcal{C}} \circ W_{*}(L)$ the $\Lambda$-homomorphism $T_{n}(L)$ becomes the identity.

We show for any $L \in \mathcal{S}$ that $T_{n}(L)$ is an isomorphism by induction over the number of vertices of $L$. If $L$ is the empty subcomplex, then $L$ is in the image of $I: \mathcal{P} \rightarrow \mathcal{S}$ and the claim has already been proved. The induction step is done as follows. We only have to consider the case, where $L$ is not in the image of $I: \mathcal{P} \rightarrow \mathcal{S}$. Since $L$ is a flag complex, there must be two vertices $v_{1}$ and $v_{2}$ in $L$ which are not connected by an edge. Let $L_{1}$ be the flag subcomplex of $L$ spanned by $v_{1}$ and all vertices in $L$ which are connected to $v_{1}$ by an edge. In particular $v_{2}$ is not a vertex of $L_{1}$. Let $L_{0}$ be the flag subcomplex of $L$ which is spanned by all vertices $v$ for which there exists an edge whose terminal points are $v$ and $v_{1}$. Notice that $v_{1}$ does not belong to $L_{0}$ and $L_{1}$ is the cone over $L_{0}$ with cone point $v_{1}$. Let $L_{2}$ be the flag subcomplex of $L$ spanned by all vertices except $v_{1}$. Then $L=L_{1} \cup L_{2}$ and $L_{0}=L_{1} \cap L_{2}$ and the number of vertices of $L_{0}, L_{1}$ and $L_{2}$ is smaller than the number of vertices of $L$. The induction hypothesis applies to $L_{k}$ and hence $T_{n}\left(L_{k}\right)$ is an isomorphism for $k=0,1$, 2. Since $\mathcal{P}(L)=\mathcal{P}\left(L_{1}\right) \cup \mathcal{P}\left(L_{2}\right)$ and $\mathcal{P}\left(L_{0}\right)=\mathcal{P}\left(L_{1}\right) \cap \mathcal{P}\left(L_{2}\right)$, the sequence induced by the inclusions $\mathcal{P}\left(L_{0}\right) \subseteq \mathcal{P}\left(L_{k}\right)$ and $\mathcal{P}\left(L_{k}\right) \subseteq \mathcal{P}(L)$ for $k=1,2$

$$
C_{n}\left(L_{0}\right) \rightarrow C_{n}\left(L_{1}\right) \oplus C_{n}\left(L_{2}\right) \rightarrow C_{n}(L) \rightarrow 0
$$

is exact. Since the group homomorphism $W_{*}\left(L_{0}\right) \rightarrow W_{*}\left(L_{1}\right)$ is split injective, a retraction is given by $W^{*}\left(L_{1}\right) \rightarrow W^{*}\left(L_{0}\right)$, the $\Lambda$-homomorphism $\mathcal{H}_{n}^{\mathcal{C}} \circ W_{*}\left(L_{0} \leq\right.$ $\left.L_{1}\right): \mathcal{H}_{n}^{\mathcal{C}} \circ W_{*}\left(L_{0}\right) \rightarrow \mathcal{H}_{n}^{\mathcal{C}} \circ W_{*}\left(L_{1}\right)$ is split injective by functoriality. We get from Lemma 2.5 and Lemma 3.1 (i) an exact sequence

$$
\mathcal{H}_{n}^{\mathcal{C}} \circ W_{*}\left(L_{0}\right) \rightarrow \mathcal{H}_{n}^{\mathcal{C}} \circ W_{*}\left(L_{1}\right) \oplus \mathcal{H}_{n}^{\mathcal{C}} \circ W_{*}\left(L_{2}\right) \rightarrow \mathcal{H}_{n}^{\mathcal{C}} \circ W_{*}(L) \rightarrow 0 .
$$

One easily checks that we obtain a commutative diagram with exact rows

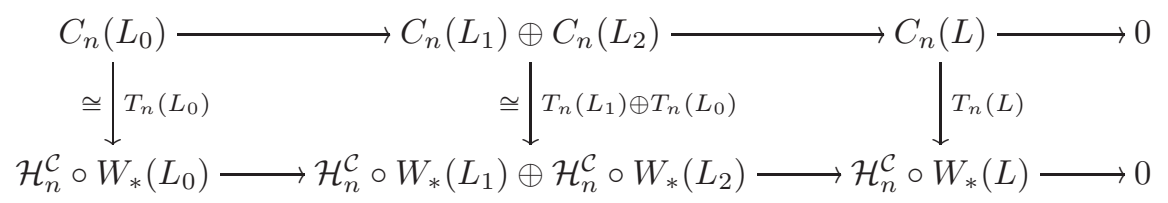

Now the induction step follows from the Five-Lemma.

We have proven part (i) of the main Theorem 1.1. 


\section{Mackey modules}

In this section we prove the remaining parts of the main Theorem 1.1. More generally, we show that the colimit of any Mackey module splits as a direct sum over its index category.

Let $\Sigma$ be a finite simplicial complex. Denote by $\mathcal{P}=\mathcal{P}(\Sigma)$ the poset of its simplices ordered by inclusion, where we allow the empty simplex as well. The dimension of the empty simplex is defined to be -1 . Notice that for two elements $\sigma$ and $\tau$ in $\mathcal{P}$ the intersection $\sigma \cap \tau$ is again an element in $\mathcal{P}$ which is uniquely determined by the property that it is maximal among all those elements $\mu$ in $\mathcal{P}$ satisfying both $\mu \leq \sigma$ and $\mu \leq \tau$.

Let $\Lambda$ be a commutative ring with unit. A Mackey $\Lambda \mathcal{P}$-module $M=\left(M_{*}, M^{*}\right)$ is a bifunctor $\mathcal{P} \rightarrow \Lambda$-Modules, i.e., a covariant functor $M_{*}$ and a contravariant functor $M^{*}$ from $\mathcal{P}$ to $\Lambda$-Modules such that $M_{*}$ and $M^{*}$ agree on objects and for objects $\sigma_{1}, \sigma_{2}, \tau$ of $\mathcal{P}$ satisfying $\sigma_{k} \leq \tau$ for $k=1,2$, we get

(4.1) $M^{*}\left(\sigma_{2} \leq \tau\right) \circ M_{*}\left(\sigma_{1} \leq \tau\right)=M_{*}\left(\sigma_{1} \cap \sigma_{2} \leq \sigma_{2}\right) \circ M^{*}\left(\sigma_{1} \cap \sigma_{2} \leq \sigma_{1}\right)$.

The name Mackey $\Lambda \mathcal{P}$-module comes from the analogy to the classical notion of a Mackey functor, where (4.1) replaces the double coset formula, see [37, Section 6.1]. for an object $\tau$ of $\mathcal{P}$.

Example 4.2 (Mackey modules coming from graph products). Our main example comes from Section 3. Let $X$ be a finite simplicial graph on the vertex set $V$ and suppose that we are given a collection of groups $\mathcal{W}:=\left\{W_{v} \mid v \in V\right\}$. Let $F:$ Groups $\rightarrow \Lambda$-Modules be a covariant functor, e.g. the functor $\mathcal{H}_{*}^{\mathcal{C}}$ defined in (2.4). Define $M_{*}=F \circ W_{*} \circ I$ and $M^{*}=F \circ W^{*} \circ I$. Then the pair $\left(M_{*}, M^{*}\right)$ defines a Mackey $\Lambda \mathcal{P}$-module by Lemma 3.1 (ii).

Fix elements $\tau$ in $\mathcal{P}$ and $d \in\{-2,-1,0,1,2, \ldots\}$. Consider a covariant $\Lambda \mathcal{P}$ module $N$, i.e., a covariant functor $N: \mathcal{P} \rightarrow \Lambda$-Modules. Define the $\Lambda$-submodules $L_{\tau}^{d} N$ and $L_{\tau} N$ of $N(\tau)$ to be the images of the maps

$$
\bigoplus_{\substack{\sigma \in \mathcal{P}, \sigma<\tau \\ \operatorname{dim}(\sigma) \leq d}} N(\sigma \leq \tau): \bigoplus_{\substack{\sigma \in \mathcal{P}, \sigma<\tau \\ \operatorname{dim}(\sigma) \leq d}} N(\sigma) \rightarrow N(\tau)
$$

and

$$
\bigoplus_{\sigma \in \mathcal{P}, \sigma<\tau} N(\sigma \leq \tau): \bigoplus_{\sigma \in \mathcal{P}, \sigma<\tau} N(\sigma) \rightarrow N(\tau),
$$

respectively. Define $\Lambda$-quotient modules of $N(\tau)$ by

$$
\begin{aligned}
& S_{\tau}^{d} N=N(\tau) / L_{\tau}^{d}(N) ; \\
& S_{\tau} N=N(\tau) / L_{\tau}(N) .
\end{aligned}
$$

Then we obtain a sequence of inclusions of $\Lambda$-modules

$$
\{0\}=L_{\tau}^{-2} N \subseteq L_{\tau}^{-1} N \subseteq L_{\tau}^{0} N \subseteq L_{\tau}^{1} N \subseteq \cdots \subseteq L_{\tau}^{\operatorname{dim}(\tau)-1} N=L_{\tau} N,
$$

and a sequence of epimorphisms of $\Lambda$-modules

$$
N(\tau)=S_{\tau}^{-2} N \rightarrow S_{\tau}^{-1} N \rightarrow S_{\tau}^{0} N \rightarrow S_{\tau}^{1} N \rightarrow \cdots \rightarrow S_{\tau}^{\operatorname{dim}(\tau)-1} N=S_{\tau} N .
$$

Note that $L_{\tau}^{-1} N=\operatorname{im}(N(\emptyset) \rightarrow N(\tau))$ and $S_{\tau}^{-1} N=\operatorname{cok}(N(\emptyset) \rightarrow N(\tau))$ for $\tau \neq \emptyset$ and $L_{\emptyset}^{-1} N=\{0\}$ and $S_{\emptyset}^{-1} N=N(\emptyset)$.

Consider a Mackey $\Lambda \mathcal{P}$-module $M=\left(M_{*}, M^{*}\right)$. Define a $\Lambda$-homomorphism

$$
s_{\tau}^{d}: M_{*}(\tau) \rightarrow M_{*}(\tau)
$$


by

$$
s_{\tau}^{d}:=\operatorname{id}_{M_{*}(\tau)}-\sum_{\substack{\mu \in \mathcal{P}, \mu<\tau \\ \operatorname{dim}(\mu)=d}} M_{*}(\mu \leq \tau) \circ M^{*}(\mu \leq \tau) .
$$

Lemma 4.3. We have for $d \in\{-1,0,1,2, \ldots\}$

$$
s_{\tau}^{d}\left(L_{\tau}^{d} M_{*}\right) \subseteq L_{\tau}^{d-1} M_{*} .
$$

Proof. We compute for $\sigma \in \mathcal{P}$ satisfying $\sigma<\tau$ and $\operatorname{dim}(\sigma) \leq d$

$$
\begin{aligned}
& s_{\tau}^{d} \circ M_{*}(\sigma \leq \tau) \\
& =M_{*}(\sigma \leq \tau)-\sum_{\substack{\mu \in \mathcal{P}, \mu<\tau \\
\operatorname{dim}(\mu)=d}} M_{*}(\mu \leq \tau) \circ M^{*}(\mu \leq \tau) \circ M_{*}(\sigma \leq \tau) \\
& \stackrel{(4.1)}{=} M_{*}(\sigma \leq \tau)-\sum_{\substack{\mu \in \mathcal{P}, \mu<\tau \\
\operatorname{dim}(\mu)=d}} M_{*}(\mu \leq \tau) \circ M_{*}(\mu \cap \sigma \leq \mu) \circ M^{*}(\mu \cap \sigma \leq \sigma) \\
& =M_{*}(\sigma \leq \tau)-\sum_{\substack{\mu \in \mathcal{P}, \mu<\tau \\
\operatorname{dim}(\mu)=d}} M_{*}(\mu \cap \sigma \leq \tau) \circ M^{*}(\mu \cap \sigma \leq \sigma) \\
& =M_{*}(\sigma \leq \tau)-\sum_{\substack{\mu \in \mathcal{P}, \mu<\tau \\
\operatorname{dim}(\mu)=d, \operatorname{dim}(\mu \cap \sigma)=d}} M_{*}(\mu \cap \sigma \leq \tau) \circ M^{*}(\mu \cap \sigma \leq \sigma) \\
& -\sum_{\substack{\mu \in \mathcal{P}, \mu<\tau \\
\operatorname{dim}(\mu)=d, \operatorname{dim}(\mu \cap \sigma) \leq d-1}} M_{*}(\mu \cap \sigma \leq \tau) \circ M^{*}(\mu \cap \sigma \leq \sigma) .
\end{aligned}
$$

Suppose that $\operatorname{dim}(\mu \cap \sigma)=d$. Since $\operatorname{dim}(\mu)=d$ and $\operatorname{dim}(\sigma) \leq d$, we conclude $\mu=\mu \cap \sigma=\sigma$ and hence because of $\sigma<\tau$

$$
\{\mu \in \mathcal{P} \mid \mu<\tau, \operatorname{dim}(\mu)=d, \operatorname{dim}(\mu \cap \sigma)=d\}=\{\sigma\} .
$$

This implies

$$
M_{*}(\sigma \leq \tau)-\sum_{\substack{\mu \in \mathcal{P}, \mu<\tau \\ \operatorname{dim}(\mu)=d, \operatorname{dim}(\mu \cap \sigma)=d}} M_{*}(\mu \cap \sigma \leq \tau) \circ M^{*}(\mu \cap \sigma \leq \sigma)=0,
$$

and hence

$$
s_{\tau}^{d} \circ M_{*}(\sigma \leq \tau)=-\sum_{\substack{\mu \in \mathcal{P}, \mu<\tau \\ \operatorname{dim}(\mu)=d, \operatorname{dim}(\mu \cap \sigma) \leq d-1}} M_{*}(\mu \cap \sigma \leq \tau) \circ M^{*}(\mu \cap \sigma \leq \sigma) .
$$

We conclude for every $\sigma \in \mathcal{P}$ satisfying $\sigma<\tau$ and $\operatorname{dim}(\sigma) \leq d$

$$
\operatorname{im}\left(s_{\tau}^{d} \circ M_{*}(\sigma \leq \tau)\right) \subseteq L_{\tau}^{d-1} M_{*} .
$$

Now the assertion follows.

Define a map

$$
s_{\tau}: M_{*}(\tau) \rightarrow M_{*}(\tau)
$$

to be the composite $s_{\tau}^{-1} \circ s_{\tau}^{0} \circ \cdots \circ s_{\tau}^{\operatorname{dim}(\tau)-1}$. Then we conclude by induction from Lemma 4.3 that $s_{\tau}$ restricted to $L_{\tau} M_{*}$ is trivial and hence induces a $\Lambda$ homomorphism

$$
\bar{s}_{\tau}: S_{\tau} M_{*} \rightarrow M_{*}(\tau) .
$$


Lemma 4.4. Let $M=\left(M_{*}, M^{*}\right)$ be a Mackey $\Lambda \mathcal{P}$-module. Consider an element $\tau \in \mathcal{P}$. Let $p_{\tau}: M_{*}(\tau) \rightarrow S_{\tau} M_{*}$ be the projection. Then

$$
p_{\tau} \circ \bar{s}_{\tau}=\operatorname{id}_{S_{\tau} M_{*}} .
$$

Proof. Obviously each map $s_{\tau}^{d}$ satisfies $p_{\tau} \circ s_{\tau}^{d}=p_{\tau}$.

For every element $\tau \in \mathcal{P}$ the restriction

$$
\mathrm{RES}_{\tau}: \Lambda \mathcal{P} \text {-Modules } \rightarrow \Lambda \text {-Modules }
$$

has a left adjoint $E_{\tau}$ by [23, Lemma 9.31 on page 171]. Explicitly, the functor $E_{\tau}: \Lambda$-Modules $\rightarrow \Lambda \mathcal{P}$-Modules is given by $E_{\tau}(N)=N \otimes_{\Lambda} \Lambda \operatorname{mor}_{\mathcal{P}}(\tau, ?)$, where ? runs through the objects of $\mathcal{P}$ and $\Lambda \operatorname{mor}_{\mathcal{P}}(\tau$, ?) is the free $\Lambda$-module with the set $\operatorname{mor}_{\mathcal{P}}(\tau, ?)$ as basis. Equivalently, $N \otimes_{\Lambda} \Lambda \operatorname{mor}_{\mathcal{P}}(\tau, ?)$ assigns to an object ? the $\Lambda$-module $N$ if $\tau \leq$ ?, and $\{0\}$ otherwise. Functoriality in ? is given by the identity on $\{0\}$ or $N$, or by the inclusion $\{0\} \rightarrow N$.

We get for every element $\tau \in \mathcal{P}$ and every map of covariant $\Lambda$-modules $u: S_{\tau} M_{*} \rightarrow$ $M_{*}(\tau)$ a map of covariant $\Lambda \mathcal{P}$-modules

$$
\operatorname{ad}(u): E_{\tau}\left(S_{\tau} M_{*}\right) \rightarrow M_{*}
$$

by the adjoint of $u$ under the adjunction $\left(E_{\tau}, \operatorname{RES}_{\tau}\right)$. For $? \in \mathcal{P}$ the $\operatorname{map} \operatorname{ad}(u)(?)$ is given by the composite $S_{\tau} M_{*} \stackrel{u}{\rightarrow} M_{*}(\tau) \stackrel{M_{*}(\tau \leq ?)}{\longrightarrow} M_{*}(?)$ if $\tau \leq$ ? and by the inclusion $\{0\} \rightarrow M_{*}(?)$ otherwise.

Lemma 4.5. Consider any collection of homomorphisms $\widehat{s}_{\tau}: S_{\tau} M_{*} \rightarrow M_{*}(\tau)$ satisfying $p_{\tau} \circ \widehat{s}_{\tau}=\mathrm{id}_{S_{\tau} M_{*}}$, where $\tau$ runs through the elements in $\mathcal{P}$.

Then the homomorphism of covariant $\Lambda \mathcal{P}$-modules

$$
\operatorname{ad}(\widehat{s}):=\bigoplus_{\tau \in \mathcal{P}} \operatorname{ad}\left(\widehat{s}_{\tau}\right): \bigoplus_{\tau \in \mathcal{P}} E_{\tau}\left(S_{\tau} M_{*}\right) \rightarrow M_{*}
$$

is an isomorphism.

Proof. We start with injectivity. Suppose that ad $(\widehat{s})$ is not injective. Then there exists an element $\sigma \in \mathcal{P}$ and a non-trivial element $a=\left(a_{\tau}\right)_{\tau \in \mathcal{P}} \in \bigoplus_{\tau \in \mathcal{P}} E_{\tau}\left(S_{\tau} M_{*}\right)(\sigma)$ such that $\operatorname{ad}(\widehat{s})(\sigma)(a)=0$. Choose $\tau_{0} \in \mathcal{P}$ with $a_{\tau_{0}} \neq 0$ such that for all $\tau \in \mathcal{P}$ with $a_{\tau} \neq 0$ we have $\operatorname{dim}(\tau) \leq \operatorname{dim}\left(\tau_{0}\right)$. If $\tau \in \mathcal{P}$ satisfies $a_{\tau} \neq 0$ and hence $E_{\tau}\left(S_{\tau} M_{*}\right)(\sigma) \neq 0$, we use the explicit description of $E_{\tau}\left(S_{\tau} M_{*}\right)$ to conclude $\tau \leq \sigma$. The composite

$$
\bigoplus_{\tau \in \mathcal{P}} E_{\tau}\left(S_{\tau} M_{*}\right)(\sigma) \stackrel{\operatorname{ad}(\widehat{s})(\sigma)}{\longrightarrow} M_{*}(\sigma) \stackrel{M^{*}\left(\tau_{0} \leq \sigma\right)}{\longrightarrow} M_{*}\left(\tau_{0}\right) \stackrel{p_{\tau_{0}}}{\longrightarrow} S_{\tau_{0}} M_{*}
$$

sends $a$ to 0 since $\operatorname{ad}(\widehat{s})(\sigma)(a)=0$.

Consider an element $\tau \in \mathcal{P}$ with $a_{\tau} \neq 0$. Then $\operatorname{dim}(\tau) \leq \operatorname{dim}\left(\tau_{0}\right)$ and hence we get $\tau \cap \tau_{0}<\tau_{0}$ if $\tau \neq \tau_{0}$ and $\tau \cap \tau_{0}=\tau_{0}$ if $\tau=\tau_{0}$. The composite

$$
M_{*}(\tau) \stackrel{M_{*}(\tau \leq \sigma)}{\longrightarrow} M_{*}(\sigma) \stackrel{M^{*}\left(\tau_{0} \leq \sigma\right)}{\longrightarrow} M_{*}\left(\tau_{0}\right) \stackrel{p_{\tau_{0}}}{\longrightarrow} S_{\tau_{0}} M_{*}
$$

agrees because of (4.1) with the composite

$$
M_{*}(\tau) \stackrel{M^{*}\left(\tau \cap \tau_{0} \leq \tau\right)}{\longrightarrow} M_{*}\left(\tau \cap \tau_{0}\right) \stackrel{M_{*}\left(\tau \cap \tau_{0} \leq \tau_{0}\right)}{\longrightarrow} M_{*}\left(\tau_{0}\right) \stackrel{p_{\tau_{0}}}{\longrightarrow} S_{\tau_{0}} M_{*} .
$$

Hence it is zero if $\tau \neq \tau_{0}$ and it is $p_{\tau_{0}}$ if $\tau=\tau_{0}$. This implies that the restriction of the composite (4.6) to the summand associated to $\tau$ is trivial if $\tau \neq \tau_{0}$ and is the identity under the obvious identification $E_{\tau_{0}}\left(S_{\tau_{0}} M_{*}\right)(\sigma)=S_{\tau_{0}} M_{*}$ if $\tau=\tau_{0}$. We conclude that the composite (4.6) sends $a$ to $a_{\tau_{0}}$ under the obvious identification $E_{\tau_{0}}\left(S_{\tau_{0}} M_{*}\right)(\sigma)=S_{\tau_{0}} M_{*}$. Since this implies $a_{\tau_{0}}=0$, we get a contradiction. This finishes the proof that $\operatorname{ad}(\widehat{s})$ is injective. 
Next we show by induction for $d=-1,0,1,2, \ldots$ that $\operatorname{ad}(\widehat{s})(\sigma)$ is surjective for all $\sigma \in \mathcal{P}$ with $\operatorname{dim}(\sigma) \leq d$. The induction beginning is obvious since $\emptyset$ is the unique initial object, hence $S_{\emptyset} M_{*}=M_{*}(\emptyset)$ holds and therefore $\operatorname{ad}(\widehat{s})_{\emptyset}(\emptyset)$ is bijective. The induction step from $(d-1)$ to $d \geq 0$ is done as follows. The composite

$$
E_{\sigma}\left(S_{\sigma} M_{*}\right)(\sigma) \stackrel{\operatorname{ad}(\widehat{s})_{\sigma}}{\longrightarrow} M_{*}(\sigma) \rightarrow S_{\sigma} M_{*}
$$

is surjective. Hence it suffices to show that $L_{\sigma} M_{*}$ is contained in the restriction of $\operatorname{ad}(\widehat{s})(\sigma)$ to $\bigoplus_{\tau \in \mathcal{P}, \tau \neq \sigma} E_{\tau}\left(S_{\tau} M_{*}\right)(\sigma)$. It suffices to show that for every $\sigma^{\prime}$ with $\sigma^{\prime}<\sigma$ the image of $M_{*}\left(\sigma^{\prime}\right) \rightarrow M_{*}(\sigma)$ is contained in the restriction of $\operatorname{ad}(\widehat{s})(\sigma)$ to $\bigoplus_{\tau \in \mathcal{P}, \tau \neq \sigma} E_{\tau}\left(S_{\tau} M_{*}\right)(\sigma)$. By induction hypothesis $\operatorname{ad}(\widehat{s})\left(\sigma^{\prime}\right)$ is surjective. Now Lemma 4.5 follows from naturality of $\operatorname{ad}(\widehat{s})$ and the fact that $E_{\sigma}\left(S_{\sigma} M_{*}\right)\left(\sigma^{\prime}\right)$ vanishes.

For a covariant $\Lambda \mathcal{P}$-module $N$, denote by $H_{n}(\mathcal{P} ; N)$ its homology. This is $H_{n}\left(P_{*} \otimes_{\Lambda \mathcal{P}} N\right)$ for the $\Lambda$-homology of the $\Lambda$-chain complex $P_{*} \otimes_{\Lambda \mathcal{P}} N$ for any projective $\Lambda \mathcal{P}$-resolution $P_{*}$ of the constant $\Lambda \mathcal{P}$-module $\underline{\Lambda}$ with value $\Lambda$. In the notation of [23, Chapter 17] this is $\operatorname{Tor}_{n}^{\Lambda \mathcal{P}}(\underline{\Lambda}, N)$.

Theorem 4.7. Let $M=\left(M_{*}, M^{*}\right)$ be a Mackey $\Lambda \mathcal{P}$-module. Then

(i) $H_{n}\left(\mathcal{P} ; M_{*}\right)$ vanishes for $n \geq 1$;

(ii) We obtain an isomorphism

$$
\operatorname{colim}_{\mathcal{P}} M_{*} \cong H_{0}\left(\mathcal{P} ; M_{*}\right) \cong \bigoplus_{\sigma \in \mathcal{P}} S_{\sigma} M_{*} ;
$$

(iii) $S_{\sigma} M_{*}$ is a direct summand in the $\Lambda$-module $M_{*}(\sigma)$.

Proof. (i) From Lemma 4.5, we obtain an isomorphism

$$
\bigoplus_{\sigma \in \mathcal{P}} H_{n}\left(\mathcal{P} ; E_{\sigma}\left(S_{\sigma} M_{*}\right)\right) \cong H_{n}\left(\mathcal{P} ; \bigoplus_{\sigma \in \mathcal{P}} E_{\sigma}\left(S_{\sigma} M_{*}\right)\right) \cong H_{n}\left(\mathcal{P} ; M_{*}\right)
$$

Since the automorphism group of the object $\sigma$ in $\mathcal{P}$ is the trivial group $\{1\}$, we get for any $\Lambda$-module $N$ an isomorphism

$$
H_{n}\left(\mathcal{P} ; E_{\sigma}(N)\right) \cong H_{n}(\{1\} ; N) \cong \begin{cases}\{0\} & \text { if } n \geq 1 ; \\ N & \text { if } n=0 .\end{cases}
$$

This follows from the adjunction $\left(E_{\sigma}, \mathrm{RES}_{\sigma}\right)$ of [23, Lemma 9.31 on page 171] and the fact that $\operatorname{RES}_{\sigma}\left(P_{*}\right)$ is a projective $\Lambda$-resolution of $\Lambda$.

(ii) For every covariant $\Lambda \mathcal{P}$-module $N$, there are canonical $\Lambda$-isomorphisms

$$
\operatorname{colim}_{\mathcal{P}} N \cong \underline{\Lambda} \otimes_{\Lambda \mathcal{P}} N \cong H_{0}(\mathcal{P} ; N),
$$

where $\underline{\Lambda}$ is the constant $\Lambda \mathcal{P}$-module with value $\Lambda$. This follows from the adjunction between tensor product and the hom-functor and the fact that $-\otimes_{\Lambda \mathcal{P}} N$ is rightexact, see [23, 9.21 and 9.23 on page 169].

(iii) This follows from Lemma 4.4 .

Let $\operatorname{ch}_{n}(\Sigma, \sigma)$ be the set of $n$-chains $\sigma_{0}<\sigma_{1}<\cdots<\sigma_{n}$ in $\mathcal{P}$ with $\sigma_{0}=\sigma$. Define the integer

$$
n_{\sigma}:=\sum_{n \geq 0}(-1)^{n} \cdot\left|\operatorname{ch}_{n}(\Sigma, \sigma)\right| .
$$

Fix a class of $\Lambda$-modules $\mathcal{E}$ with the property that for an exact sequence $0 \rightarrow$ $V_{0} \rightarrow V_{1} \rightarrow V_{2} \rightarrow 0$ the $\Lambda$-module $V_{1}$ belongs to $\mathcal{E}$ if and only if both $V_{0}$ and $V_{2}$ belong to $\mathcal{E}$. An example is the class $\mathcal{E}$ of $\Lambda$-modules whose underlying set is finite, and for a Noetherian ring $\Lambda$ the class $\mathcal{E}$ of finitely generated $\Lambda$-modules. Denote by $G_{0}(\mathcal{E})$ the Grothendieck group of elements in $\mathcal{E}$, i.e., the abelian group with the isomorphism classes of elements in $\mathcal{E}$ as generators and relations $\left[V_{1}\right]=\left[V_{0}\right]+\left[V_{2}\right]$ 
for every short exact sequence $0 \rightarrow V_{0} \rightarrow V_{1} \rightarrow V_{2} \rightarrow 0$ of $\Lambda$-modules belonging to $\mathcal{E}$.

Theorem 4.8. Let $M=\left(M_{*}, M^{*}\right)$ be a Mackey $\Lambda \mathcal{P}$-module. Suppose that $M_{*}(\sigma)$ lies in $\mathcal{E}$ for all $\sigma \in \mathcal{P}$.

Then we get in $G_{0}(\mathcal{E})$

$$
\left[\operatorname{colim}_{\mathcal{P}} M_{*}\right]=\sum_{\sigma \in \mathcal{P}} n_{\sigma} \cdot\left[M_{*}(\sigma)\right] .
$$

Proof. The bar-resolution yields a finite free $\Lambda \mathcal{P}$-resolution $C_{*}$ of the constant $\Lambda \mathcal{P}$ module $\underline{\Lambda}$ with value $\Lambda$ such that

$$
C_{n}=\bigoplus_{\sigma \in \mathcal{P}} \bigoplus_{\operatorname{ch}_{n}(\Sigma, \sigma)} \Lambda \operatorname{mor}_{\mathcal{P}}(?, \sigma),
$$

see [11, Section 3]. Since $C_{*}$ is a finite free $\Lambda \mathcal{P}$-chain complex, the $\Lambda$-chain complex $C_{*} \otimes_{\Lambda \mathcal{P}} M_{*}$ is a finite-dimensional $\Lambda$-complex whose $\Lambda$-chain modules belong to $\mathcal{E}$, and we get in $G_{0}(\mathcal{E})$

$$
\begin{aligned}
\sum_{n \geq 0}(-1)^{n} \cdot\left[H_{n}\left(C_{*} \otimes_{\Lambda \mathcal{P}} M_{*}\right)\right] & =\sum_{n \geq 0}(-1)^{n} \cdot\left[C_{n} \otimes_{\Lambda \mathcal{P}} M_{*}\right] \\
& =\sum_{n \geq 0}(-1)^{n} \cdot\left(\sum_{\sigma \in \mathcal{P}} \sum_{\operatorname{ch}_{n}(\Sigma, \sigma)}\left[M_{*}(\sigma)\right]\right) \\
& =\sum_{\sigma \in \mathcal{P}}\left(\sum_{n \geq 0}(-1)^{n} \cdot\left|\operatorname{ch}_{n}(\Sigma, \sigma)\right|\right) \cdot\left[M_{*}(\sigma)\right] \\
& =\sum_{\sigma \in \mathcal{P}} n_{\sigma} \cdot\left[M_{*}(\sigma)\right] .
\end{aligned}
$$

Since $H_{n}\left(C_{*} \otimes_{\Lambda \mathcal{P}} M_{*}\right)$ agrees with $H_{n}\left(\mathcal{P} ; M_{*}\right)$, the claim follows from Theorem 4.7.

4.1. Proof of Theorem 1.1. We defined the functor $\mathcal{H}_{n}^{\mathcal{C}}$ : Groups $\rightarrow \Lambda$-Modules in (2.4). We conclude from Example 4.2 that $\left(\mathcal{H}_{n}^{\mathcal{C}} \circ W_{*} \circ I, \mathcal{H}_{n}^{\mathcal{C}} \circ W^{*} \circ I\right)$ is a Mackey $\Lambda \mathcal{P}$-module. Now Theorem 1.1 follows from Theorem 3.4, Theorem 4.7, and Theorem 4.8 .

\section{IsOmORPhism CONJECTURES IN $K$ - AND $L$-THEORY}

In this section we review the Isomorphism Conjectures of Baum-Cones and Farrell--Jones and recollect the most important results on the passage from $\mathcal{F} \mathcal{I} \mathcal{N}$ to $\mathcal{V C Y}$ in the Farrell--Jones setting.

Let $\mathcal{C}$ be a non-empty class of groups which is closed under isomorphisms, passage to subgroups and passage to quotient groups. Recall that given a group $G$, we denote by $E_{\mathcal{C}}(G)$ the classifying space of $G$ with respect to the family of subgroups $\mathcal{F}_{\mathcal{C}}(G)=\{H \subseteq G \mid H \in \mathcal{C}\}$. Consider a covariant Groupoids-spectrum

\section{E: Groupoids $\rightarrow$ Spectra}

which respects equivalences. We obtain an equivariant homology theory $H_{*}^{?}(-; \mathbf{E})$ associated to $\mathbf{E}$ from Theorem 2.2.

Then the Meta-Isomorphism Conjecture for $\mathbf{E}$ and the class $\mathcal{C}$ predicts that the projection pr: $E_{\mathcal{C}}(G) \rightarrow G / G$ induces for all $n \in \mathbb{Z}$ an isomorphism

$$
H_{n}^{G}(\mathrm{pr} ; \mathbf{E}): H_{n}^{G}\left(E_{\mathcal{C}}(G) ; \mathbf{E}\right) \stackrel{\cong}{\longrightarrow} H_{n}^{G}(G / G ; \mathbf{E})=\pi_{n}(\mathbf{E}(G)) .
$$

If we make the appropriate choices for $\mathbf{E}$ and $\mathcal{C}$, this becomes the Baum-Connes Conjecture or it becomes the Farrell-Jones Conjecture for algebraic $K$-theory, for 
algebraic $L$-theory, for Waldhausen's $A$-theory, or for topological Hochschild homology.

5.1. The Baum-Connes Conjecture. Given a discrete group $G$, denote by $C_{r}^{*}(G)$ and $C_{r}^{*}(G ; \mathbb{R})$ its reduced complex and reduced real group $C^{*}$-algebra and by $C_{m}^{*}(G)$ and $C_{m}^{*}(G ; \mathbb{R})$ its maximal complex and maximal real group $C^{*}$-algebra. There are covariant functors

$$
\begin{aligned}
\mathbf{K}_{C_{m}^{*}}: \text { Groupoids } & \rightarrow \text { Spectra } \\
\mathbf{K}_{C_{m}^{*} ; \mathbb{R}}: \text { Groupoids } & \rightarrow \text { Spectra },
\end{aligned}
$$

which send equivalences of groupoids to weak equivalences of spectra and satisfy $\pi_{n}\left(\mathbf{K}_{C_{m}^{*}}(G)\right)=K_{n}\left(C_{m}^{*}(G)\right)$ and $\pi_{n}\left(\mathbf{K}_{C_{m}^{*} ; \mathbb{R}}(G)\right)=K O_{n}\left(C_{m}^{*}(G ; \mathbb{R})\right)$ for $n \in \mathbb{Z}$. Here $K_{*}$ and $K O_{*}$ denote topological $K$-theory. If we consider the class of finite groups, the Meta-Isomorphism Conjecture reduces to the Baum-Connes Conjecture for the maximal group $C^{*}$-algebra. It predicts the bijectivity of the assembly maps for $n \in \mathbb{Z}$

$$
\begin{aligned}
K_{n}^{G}(\underline{E} G) & \rightarrow K_{n}\left(C_{m}^{*}(G)\right) \\
K O_{n}^{G}(\underline{E} G) & \rightarrow K O_{n}\left(C_{m}^{*}(G ; \mathbb{R})\right),
\end{aligned}
$$

where the source is given by equivariant $K$-homology for which we have the identifications $K_{n}^{G}(\underline{E} G)=H_{n}^{G}\left(\underline{E} G ; \mathbf{K}_{C_{m}^{*}}\right)$ and $K O_{n}^{G}(\underline{E} G)=H_{n}^{G}\left(\underline{E} G ; \mathbf{K}_{C_{m}^{*} ; \mathbb{R}}\right)$.

We can apply $K$-theory to the natural maps of $C^{*}$-algebras $C_{m}^{*}(G) \rightarrow C_{r}^{*}(G)$ and $C_{m}^{*}(G ; \mathbb{R}) \rightarrow C_{r}^{*}(G ; \mathbb{R})$ to obtain maps $f_{n}: K_{n}\left(C_{m}^{*}(G)\right) \rightarrow K_{n}\left(C_{r}^{*}(G)\right)$ and $f_{n}^{\mathbb{R}}: K O_{n}\left(C_{m}^{*}(G ; \mathbb{R})\right) \rightarrow K O_{n}\left(C_{r}^{*}(G ; \mathbb{R})\right)$. The Baum-Connes Conjecture predicts that the composites

$$
\begin{gathered}
K_{n}^{G}(\underline{E} G) \rightarrow K_{n}\left(C_{m}^{*}(G)\right) \stackrel{f_{n}}{\longrightarrow} K_{n}\left(C_{r}^{*}(G)\right) ; \\
K O_{n}^{G}(\underline{E} G) \rightarrow K O_{n}\left(C_{m}^{*}(G ; \mathbb{R})\right) \stackrel{f_{n}^{\mathbb{R}}}{\longrightarrow} K O_{n}\left(C_{r}^{*}(G ; \mathbb{R})\right)
\end{gathered}
$$

are bijective for all $n \in \mathbb{Z}$.

There are counterexamples to the Baum-Connes Conjecture for the maximal group $C^{*}$-algebra, but no counterexamples to the Baum-Connes Conjecture are known. We want to consider the Baum-Connes Conjecture for the maximal group $C^{*}$-algebra since $K_{n}\left(C_{m}^{*}(G)\right)$ and $K O_{n}\left(C_{m}^{*}(G ; \mathbb{R})\right)$ are functorial in $G$ for all group homomorphisms, whereas $K_{n}\left(C_{r}^{*}(G)\right)$ and $K O_{n}\left(C_{r}^{*}(G ; \mathbb{R})\right)$ are functorial for injective group homomorphisms, but not in general for any group homomorphism. Moreover the covariant functors (5.1) and (5.2) are defined on Groupoids. This ensures that the induction structure is available for all group homomorphisms and not only for injective group homomorphisms as it is the case if we replace (5.1) and (5.2) by their versions for the reduced $C^{*}$-algebras. We later want to apply the induction structure also to certain split surjective group homomorphisms, see Remark 3.2.

There is a more general Baum-Connes Conjecture with coefficients, which is known to be true for a large class of groups and which has good inheritance properties. In particular, the class of groups satisfying the Baum-Connes Conjecture with coefficients is closed under taking graph products, since it is stable under finite direct products and amalgamated products, see [31] and [32].

5.2. The Farrell-Jones Conjecture. Given a ring $R$ (with involution), there are covariant functors

$$
\begin{aligned}
\mathbf{K}_{R}: \text { Groupoids } & \rightarrow \text { Spectra } \\
\mathbf{L}_{R}^{\langle-\infty\rangle}: \text { Groupoids } & \rightarrow \text { Spectra }
\end{aligned}
$$


which send equivalences of groupoids to weak equivalences of spectra and satisfy $\pi_{n}\left(\mathbf{K}_{R}(G)\right)=K_{n}(R G)$ and $\pi_{n}\left(\mathbf{L}_{R}^{\langle-\infty\rangle}(G)\right)=L_{n}^{\langle-\infty\rangle}(R G)$. Here $K_{*}$ denotes nonconnective algebraic $K$-theory and $L_{*}^{\langle-\infty\rangle}$ denotes algebraic $L$-theory with decoration $\langle-\infty\rangle$. If we consider the class of virtually cyclic groups, the Meta-Isomorphism Conjecture reduces to the $K$-theoretic or the L-theoretic Farrell-Jones Conjecture which predicts that for all $n \in \mathbb{Z}$ the corresponding map

$$
\begin{aligned}
H_{n}^{G}\left(\underline{\underline{E}} G ; \mathbf{K}_{R}\right) & \rightarrow H_{n}^{G}\left(G / G ; \mathbf{K}_{R}\right)=K_{n}(R G) ; \\
H_{n}^{G}\left(\underline{\underline{E}} G ; \mathbf{L}_{R}^{\langle-\infty\rangle}\right) & \rightarrow H_{n}^{G}\left(G / G ; \mathbf{L}_{R}^{\langle-\infty\rangle}\right)=L_{n}^{\langle-\infty\rangle}(R G)
\end{aligned}
$$

is bijective.

There is a more general Full Farrell-Jones Conjecture which allows additive $G$-categories as coefficients. It is known to be true for a large class of groups and has good inheritance properties. In particular, the class of groups satisfying the Full Farrell-Jones Conjecture is closed under taking graph products, which is a result of Gandini-Rüping [17]. There also is a version of the Farrell-Jones Conjecture for Waldhausen's $A$-theory which we will not discuss here. It satisfies similar inheritance properties as the Full Farrell--Jones Conjecture, see [15] and [38]. Also the following Theorem 5.3 (i) holds in this setting, see [6].

5.3. The passage from $\mathcal{F} \mathcal{I N}$ to $\mathcal{V} \mathcal{C Y}$. The Farrell-Jones Conjecture is more complicated than the Baum-Connes Conjecture since for the Farrell-Jones Conjecture the class of virtually cyclic groups has to be considered, whereas for the Baum-Connes Conjecture the class of finite groups suffices. Hence one has to understand the passage from $\underline{E} G$ to $\underline{\underline{E}} G$ in the Farrell-Jones setting.

\section{Theorem 5.3.}

(i) Let $G$ be any group and $R$ be a ring. Then the relative assembly maps

$$
\begin{aligned}
H_{n}^{G}\left(\underline{E} G ; \mathbf{K}_{R}\right) & \rightarrow H_{n}^{G}\left(\underline{\underline{E}} G ; \mathbf{K}_{R}\right) ; \\
H_{n}^{G}\left(\underline{E} G ; \mathbf{L}_{R}^{\langle-\infty\rangle}\right) & \rightarrow H_{n}^{G}\left(\underline{\underline{E}} G ; \mathbf{L}_{R}^{\langle-\infty\rangle}\right)
\end{aligned}
$$

are split injective for all $n \in \mathbb{Z}$;

(ii) Let $G$ be any group and $R$ be a regular ring. Then the relative assembly map

$$
H_{n}^{G}\left(\underline{E} G ; \mathbf{K}_{R}\right) \rightarrow H_{n}^{G}\left(\underline{\underline{E}} G ; \mathbf{K}_{R}\right)
$$

is rationally bijective for all $n \in \mathbb{Z}$;

(iii) Let $G$ be any group and $R$ be a regular ring. Suppose that for any finite subgroup $H \subseteq G$ its order $|H|$ is invertible in $R$. Then the relative assembly map

is bijective for all $n \in \mathbb{Z}$;

$$
H_{n}^{G}\left(\underline{E} G ; \mathbf{K}_{R}\right) \rightarrow H_{n}^{G}\left(\underline{\underline{E}} G ; \mathbf{K}_{R}\right)
$$

(iv) Let $R$ be a regular ring. Let $W=W(X, \mathcal{W})$ be a graph product and $d$ be a natural number. Suppose that for any vertex $v \in V$ the group $W_{v}$ is either torsionfree or a finite group whose order divides $d$. Then the relative assembly map

$$
H_{n}^{W}\left(\underline{E} W ; \mathbf{K}_{R}\right) \rightarrow H_{n}^{W}\left(\underline{\underline{E}} W ; \mathbf{K}_{R}\right)
$$

is bijective after inverting $d$ for all $n \in \mathbb{Z}$;

(v) Let $G$ be any group and $R$ be a ring with involution. Then the relative assembly map

$$
H_{n}^{G}\left(\underline{E} G ; \mathbf{L}_{R}^{\langle-\infty\rangle}\right) \rightarrow H_{n}^{G}\left(\underline{\underline{E}} G ; \mathbf{L}_{R}^{\langle-\infty\rangle}\right)
$$

is bijective after inverting 2 for all $n \in \mathbb{Z}$; 
(vi) Let $G$ be any group and $R$ be a ring with involution such that 2 is invertible in $R$. Then the relative assembly map

$$
H_{n}^{G}\left(\underline{E} G ; \mathbf{L}_{R}^{\langle-\infty\rangle}\right) \rightarrow H_{n}^{G}\left(\underline{\underline{E}} G ; \mathbf{L}_{R}^{\langle-\infty\rangle}\right)
$$

is bijective for all $n \in \mathbb{Z}$.

(vii) Let $R$ be a ring with involution. Let $W=W(X, \mathcal{W})$ be a graph product. Suppose that for any vertex $v \in V$ the group $W_{v}$ is either torsionfree or a finite group of odd order. Then the relative assembly map

$$
H_{n}^{W}\left(\underline{E} W ; \mathbf{L}_{R}^{\langle-\infty\rangle}\right) \rightarrow H_{n}^{W}\left(\underline{\underline{E}} W ; \mathbf{L}_{R}^{\langle-\infty\rangle}\right)
$$

is bijective for all $n \in \mathbb{Z}$.

Proof. (i) See [3] and [28].

(ii) This is proved in [28, Theorem 0.3].

(iii) See [27, Proposition 70 on page 744].

(iv) Let $\mathcal{V} \mathcal{C} \mathcal{Y}_{I}$ be the class of virtually cyclic groups of type I, i.e., groups which admit a homomorphism to $\mathbb{Z}$ with finite kernel. Then the relative assembly map

$$
H_{n}^{W}\left(E_{\mathcal{V} \mathcal{Y}}(W) ; \mathbf{K}_{R}\right) \rightarrow H_{n}^{W}\left(E_{\mathcal{V} \mathcal{Y} Y}(W) ; \mathbf{K}_{R}\right)
$$

is bijective for all $n \in \mathbb{Z}$ by [12, Theorem 1.1]. Hence it suffices to show that

$$
H_{n}^{W}\left(E_{\mathcal{F} \mathcal{I N}}(W) ; \mathbf{K}_{R}\right) \rightarrow H_{n}^{W}\left(E_{\mathcal{V} \mathcal{C}}(W) ; \mathbf{K}_{R}\right)
$$

is bijective for all $n \in \mathbb{Z}$. By the same argument as it appears in [28, Proof of Theorem 0.3 on page 370$]$ the claim is reduced to showing that for any infinite virtually cyclic subgroup $H \subseteq W$ of type $I$ we get

$$
N K_{n}\left(R K_{H} ; \phi\right)[1 / d]=0,
$$

where $K_{H} \subseteq H$ is a finite subgroup such that $H / K_{H}$ is infinite cyclic and $\phi: K_{H} \rightarrow$ $K_{H}$ is given by conjugation with an element $h \in H$ which is sent under the projection $H \rightarrow H / K_{H}$ to a generator. Any finite subgroup of $W$ is conjugated into a group $W(\sigma)$ for some simplex $\sigma$ of $\Sigma$ such that $W_{v}$ is finite for every $v \in V \cap \sigma$. (Indeed, this is obvious if $\Sigma$ is a simplex. Otherwise, we can express $W$ as an amalgamated product and use the fact that a finite subgroup of an amalgamated product is conjugated into one of the factors, see [35, Theorem 8 in 4.3 on page 36]. Then conclude via induction on the number of vertices.) Hence we can assume that there exists a simplex $\sigma$ of $\Sigma$ such that $K_{H} \subseteq W(\sigma)$ and $W(\sigma)$ is finite. There is a group homomorphism $r: W \rightarrow W(\sigma)$ whose restriction to $W(\sigma)$ is the identity, see Remark 3.2. Consider $w \in K_{H}$. Then $h w h^{-1}$ belongs to $K_{H}$ again. We compute

$$
\phi(w)=h w h^{-1}=r\left(h w h^{-1}\right)=r(h) r(w) r(h)^{-1}=r(h) w r(h)^{-1} .
$$

Hence $\phi$ is given by conjugation with $r(h) \in W(\sigma)$. The order of $r(h)$ and thus also of $\phi$ divides $d$. We conclude from [28, Theorem 9.4] that $N K_{n}\left(R K_{H} ; \phi\right)[1 / d]=0$.

(v) This is proved in [25, Lemma 4.2].

(vi) The proof is analogous to the first part of the proof of (iv) using the fact that UNil-groups vanish, if $1 / 2$ is contained in $R$, see [7, Corollary 3] and that the map

$$
H_{n}^{G}\left(E_{\mathcal{F} \mathcal{I N}}(G) ; \mathbf{L}_{R}^{\langle-\infty\rangle}\right) \rightarrow H_{n}^{G}\left(E_{\mathcal{V C Y}}(G) ; \mathbf{L}_{R}^{\langle-\infty\rangle}\right)
$$

is bijective for all $n \in \mathbb{Z}$, see [25, Lemma 4.2].

(vii) Any finite subgroup of $W$ is conjugated into a group $W(\sigma)$ for some simplex $\sigma$ of $\Sigma$ such that $W_{v}$ is finite for every $v \in V \cap \sigma$. Hence every finite subgroup has odd order and thus every infinite virtually cyclic subgroup of $W$ is of type I. Now the claim follows from [25, Lemma 4.2]. This finishes the proof of Theorem 5.3. 


\section{Right-Angled Artin groups}

In this section we want to compute the group homology, the algebraic $K$ - and $L$-theory, and the topological $K$-theory of a right-angled Artin group $W$. Recall that a right-angled Artin group is a graph product $W=W(X, \mathcal{W})$ for which each of the groups $W_{v}$ is infinite cyclic. Note that $W$ is torsionfree. Right-angled Artin groups satisfy the Baum-Connes Conjecture and the Baum-Connes Conjecture for the maximal group $C^{*}$-algebra, which follows from [13] and [19]. Both the $K$ theoretic and the $L$-theoretic Farrell-Jones Conjecture are satisfied for right-angled Artin groups, see [2] and [40]. For general information about right-angled Artin groups we refer for instance to Charney [9].

In the sequel we denote by $r_{k}$ the number of $k$-simplices in $\mathcal{P}=\mathcal{P}(\Sigma)$ and put $r=|\mathcal{P}|=\sum_{k=-1}^{\operatorname{dim}(\Sigma)} r_{k}$. Recall that the empty simplex is allowed in $\mathcal{P}$ and has dimension -1 .

Let $\mathcal{K}_{*}$ be a (non-equivariant) generalized homology theory with values in $\Lambda$ modules. Let $X$ be a $C W$-complex. It follows from the axioms of a generalized homology theory that there is an isomorphism, natural in $X$

$$
B_{n}(X):=\mathcal{K}_{n}(\mathrm{pr}) \times\left(s_{n} \circ \mathcal{K}_{n}\left(\operatorname{id}_{X} \times i\right)\right): \mathcal{K}_{n}\left(X \times S^{1}\right) \stackrel{\cong}{\rightrightarrows} \mathcal{K}_{n}(X) \times \mathcal{K}_{n-1}(X),
$$

where we denote by pr: $X \times S^{1} \rightarrow X$ the projection, by $i: S^{1}=\left(S^{1}, \emptyset\right) \rightarrow\left(S^{1}\right.$, pt) the inclusion, and by $s_{n}: \mathcal{K}_{n}\left(X \times\left(S^{1},\{\mathrm{pt}\}\right)\right) \stackrel{\cong}{\longrightarrow} \mathcal{K}_{n-1}(X)$ the suspension isomorphism.

By induction over $k \geq 0$ we obtain an isomorphism

$$
B_{n}^{k}: \mathcal{K}_{n}\left(T^{k}\right) \cong \prod_{i=0}^{k} \prod_{j=1}^{\left(\begin{array}{c}
k \\
i
\end{array}\right)} \mathcal{K}_{n-i}(\mathrm{pt})
$$

where we denote by $T^{k}$ the $k$-dimensional torus $\prod_{i=1}^{k} S^{1}$. Note that $T^{0}=$ pt. For $i=1,2, \ldots, k$, let $T_{i}^{k} \subseteq T^{k}$ be the subspace consisting of elements $\left(z_{1}, z_{2}, \ldots, z_{k}\right)$ with $z_{i}=*$, where $*$ is a fixed base point in $S^{1}$. Let $j_{i}^{k}: T_{i}^{k} \rightarrow T^{k}$ be the inclusion. We will identify $T_{k}^{k}=T^{k-1}$.

Lemma 6.2. For every $k \geq 0$ and $n \in \mathbb{Z}$ there is an isomorphism

$$
c_{n}^{k}: \operatorname{cok}\left(\bigoplus_{i=1}^{k} \mathcal{K}_{n}\left(j_{i}^{k}\right): \bigoplus_{i=1}^{k} \mathcal{K}_{n}\left(T_{i}^{k}\right) \rightarrow \mathcal{K}_{n}\left(T^{k}\right)\right) \cong \mathcal{K}_{n-k}(\mathrm{pt})
$$

Its inverse is induced by the restriction of the inverse of the isomorphism $B_{n}^{k}$ of (6.1) to the factor $\mathcal{K}_{n-k}(\mathrm{pt})$ for the index $i=k$.

Proof. We use induction over $k=0,1,2, \ldots$ If $k=0$, take $c_{n}^{0}=\mathrm{id}_{\mathcal{K}_{n}(\mathrm{pt})}$. The induction step from $(k-1)$ to $k \geq 1$ is done as follows. We have the following 
commutative diagram of $\Lambda$-modules

$$
\begin{aligned}
& \bigoplus_{i=1}^{k} \mathcal{K}_{n}\left(T_{i}^{k}\right) \longrightarrow \bigoplus_{i=1}^{k} \mathcal{K}_{n}\left(j_{i}^{k}\right) \\
& \text { id } \mid \cong \\
& \mathcal{K}_{n}\left(T_{k}^{k}\right) \oplus \bigoplus_{i=1}^{k-1} \mathcal{K}_{n}\left(T_{i}^{k}\right) \\
& \left.\operatorname{id} \oplus \oplus_{i=1}^{k-1} \text { id }\right\rfloor \cong \\
& \mathcal{K}_{n}\left(T^{k-1}\right) \oplus \bigoplus_{i=1}^{k-1} \mathcal{K}_{n}\left(T_{i}^{k-1} \times S^{1}\right) \\
& \operatorname{id} \oplus \bigoplus_{i=1}^{k-1} B_{n}\left(T_{i}^{k-1}\right) \mid \cong \\
& \mathcal{K}_{n}\left(T^{k-1}\right) \oplus \bigoplus_{i=1}^{k-1}\left(\mathcal{K}_{n}\left(T_{i}^{k-1}\right) \oplus \mathcal{K}_{n-1}\left(T_{i}^{k-1}\right)\right) \\
& f\rfloor \\
& \left(\mathcal{K}_{n}\left(T^{k-1}\right) \oplus \bigoplus_{i=1}^{k-1} \mathcal{K}_{n}\left(T_{i}^{k-1}\right)\right) \\
& \oplus \\
& \bigoplus_{i=1}^{k-1} \mathcal{K}_{n-1}\left(T_{i}^{k-1}\right)
\end{aligned}
$$
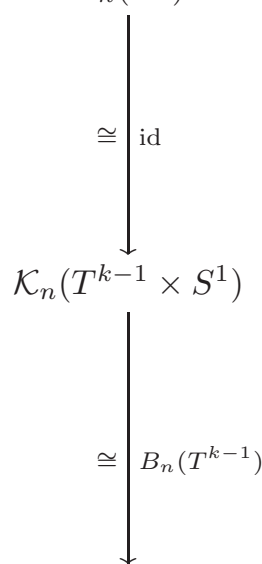

where $f$ is the obvious isomorphism, $f_{0}:=\operatorname{id}_{\mathcal{K}_{n}\left(T^{k-1}\right)} \oplus \bigoplus_{i=1}^{k-1} \mathcal{K}_{n}\left(j_{i}^{k-1}\right)$, and $f_{1}:=$ $\bigoplus_{i=1}^{k-1} \mathcal{K}_{n-1}\left(j_{i}^{k-1}\right)$. Since $f_{0}$ is surjective, the diagram above induces an isomorphism

$$
\operatorname{cok}\left(\bigoplus_{i=1}^{k} \mathcal{K}_{n}\left(j_{i}^{k}\right)\right) \stackrel{\cong}{\rightrightarrows} \operatorname{cok}\left(f_{0}\right) \oplus \operatorname{cok}\left(f_{1}\right)=\{0\} \oplus \operatorname{cok}\left(\bigoplus_{i=1}^{k-1} \mathcal{K}_{n-1}\left(j_{i}^{k-1}\right)\right) .
$$

By induction hypothesis we have an isomorphism

$$
c_{n-1}^{k-1}: \operatorname{cok}\left(\bigoplus_{i=1}^{k-1} \mathcal{K}_{n-1}\left(j_{i}^{k-1}\right)\right) \stackrel{\cong}{\rightrightarrows} \mathcal{K}_{n-k}(\mathrm{pt}) .
$$

This finishes the induction step and hence the proof of Lemma 6.2.

6.1. Group homology. Let $\mathcal{K}_{*}$ be any generalized homology theory with values in $\Lambda$-modules. Notice that for any group $G$ the $C W$-complex $E G \times{ }_{G} \underline{E} G$ is a model for $B G$ since $\underline{E} G$ is contractible after forgetting the $G$-action. We have introduced the equivariant homology theory given by the Borel construction and $\mathcal{K}_{*}$ in Example 2.3. We conclude from Theorem 1.1 and Lemma 6.2 that there is an explicit $\Lambda$-isomorphism

$$
\bigoplus_{\sigma \in \mathcal{P}} \mathcal{K}_{n-\operatorname{dim}(\sigma)-1}(\mathrm{pt}) \stackrel{\cong}{\rightrightarrows} \mathcal{K}_{n}(B W) .
$$

If we take for $\mathcal{K}_{*}$ singular homology $H_{*}(-; \Lambda)$ with coefficients in $\Lambda$, this boils down to the well-known, see for example [21, Corollary 11], isomorphism of $\Lambda$ modules

$$
\Lambda^{r_{n-1}} \stackrel{\cong}{\rightrightarrows} H_{n}(B W ; \Lambda) .
$$

In particular we get the following relation for the Euler characteristics

$$
\chi(B W)=1-\chi(\Sigma) .
$$


6.2. Algebraic $K$-theory. Let $R$ be a regular ring. We conclude from Theorem 1.1, Theorem 5.3 (iv), and Lemma 6.2 that there is an explicit isomorphism of abelian groups

$$
\bigoplus_{\sigma \in \mathcal{P}} K_{n-\operatorname{dim}(\sigma)-1}(R) \stackrel{\cong}{\longrightarrow} K_{n}(R W) .
$$

Its restriction to the summand belonging to $\sigma$ is the composite of the map $K_{n}(R W(\sigma)) \rightarrow K_{n}(R W)$ coming from the inclusion $\mathbb{Z}^{\operatorname{dim}(\sigma)+1}=W(\sigma) \rightarrow W$ with the restriction of the inverse of the iterated Bass-Heller-Swan isomorphism

$$
\bigoplus_{i=0}^{\operatorname{dim}(\sigma)+1} \bigoplus_{j=1}^{\left(\begin{array}{c}
\operatorname{dim}(\sigma)+1 \\
i
\end{array}\right.} K_{n-i}(R) \cong K_{n}\left(R\left[\mathbb{Z}^{\operatorname{dim}(\sigma)+1}\right]\right)
$$

to the summand $K_{n-\operatorname{dim}(\sigma)-1}(R)$ belonging to $i=\operatorname{dim}(\sigma)+1$.

Since for a regular ring $R$ its negative $K$-theory vanishes, we conclude $K_{n}(R W)=$ 0 for $n \leq-1$. If we take $R=\mathbb{Z}$, we conclude that $K_{n}(\mathbb{Z} W)$ for $n \leq-1, \widetilde{K}_{0}(\mathbb{Z} W)$, and $\operatorname{Wh}(W)$ vanish what is actually true if we replace $W$ by any torsionfree group satisfying the Farrell-Jones Conjecture.

6.3. Algebraic $L$-theory. Let $R$ be a ring with involution. We conclude from Theorem 1.1, Theorem 5.3 (vii), and Lemma 6.2 that there is an explicit isomorphism of abelian groups

$$
\bigoplus_{\sigma \in \mathcal{P}} L_{n-\operatorname{dim}(\sigma)-1}^{\langle-\infty\rangle}(R) \stackrel{\cong}{\longrightarrow} L_{n}^{\langle-\infty\rangle}(R W)
$$

Its restriction to a summand comes from the Shaneson splitting.

6.4. Topological $K$-theory. We conclude from Theorem 1.1 and Lemma 6.2 that there are explicit isomorphisms of abelian groups

$$
\begin{aligned}
\bigoplus_{\sigma \in \mathcal{P}} K_{n-\operatorname{dim}(\sigma)-1}(\mathbb{C}) & \cong K_{n}\left(C_{m}^{*}(W)\right) \cong K_{n}\left(C_{r}^{*}(W)\right) ; \\
\bigoplus_{\sigma \in \mathcal{P}} K O_{n-\operatorname{dim}(\sigma)-1}(\mathbb{R}) & \cong K O_{n}\left(C_{m}^{*}(W ; \mathbb{R})\right) \cong K O_{n}\left(C_{r}^{*}(W ; \mathbb{R})\right) .
\end{aligned}
$$

In particular we get an isomorphism of abelian groups

$$
K_{n}\left(C_{m}^{*}(W)\right) \cong K_{n}\left(C_{r}^{*}(W)\right) \cong \mathbb{Z}^{t_{n}}
$$

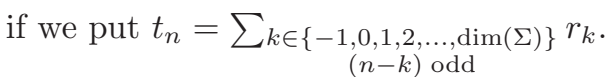

\section{Right-Angled Coxeter Groups}

In this section we want to compute the group homology, the algebraic $K$ - and $L$-theory, and the topological $K$-theory of a right-angled Coxeter group $W$. Recall that a right-angled Coxeter group is a graph product $W=W(X, \mathcal{W})$ for which each of the groups $W_{v}$ is cyclic of order two. Right-angled Coxeter groups satisfy the Baum-Connes Conjecture and the Baum-Connes Conjecture for the maximal group $C^{*}$-algebra, which follows from [13] and [19]. Both the $K$-theoretic and the $L$-theoretic Farrell-Jones Conjecture are satisfied for right-angled Coxeter groups, see [2] and [40].

In the sequel we denote by $r_{k}$ the number of $k$-simplices in $\mathcal{P}=\mathcal{P}(\Sigma)$ and put $r=|\mathcal{P}|=\sum_{k=-1}^{\operatorname{dim}(\Sigma)} r_{k}$. Recall that the empty simplex is allowed in $\mathcal{P}$ and has dimension -1 .

During this section we denote by $C_{2}$ the cyclic group of order two. Fix an integer $k \geq 1$. We will identify $C_{2}^{k}=C_{2}^{k-1} \times C_{2}$ and put $C_{2}^{0}=\{1\}$. For $i=1,2, \ldots, k$, let 
$\left(C_{2}^{k}\right)_{i}$ be the subgroup of $C_{2}^{k}$ consisting of those elements $\left(a_{1}, a_{2}, \ldots, a_{k}\right)$ satisfying $a_{i}=0$ and denote by $j_{i}^{k}:\left(C_{2}^{k}\right)_{i} \rightarrow C_{2}^{k}$ the inclusion.

7.1. Group homology. Define for $n \geq 0$ and $k \geq 0$ an integer

$$
\rho_{n, k}:=\sum_{j=k}^{n-1}(-1)^{n-1-j} \cdot\left(\begin{array}{l}
j \\
k
\end{array}\right)
$$

where here and in the sequel we use the convention $\sum_{j=a}^{b} c_{j}=0$ for $a>b$.

Theorem 7.1. We have for $n \geq 1$

$$
H_{n}(W ; \mathbb{Z}) \cong \bigoplus_{\sigma \in \mathcal{P}} \bigoplus_{j=1}^{\rho_{n, \operatorname{dim}(\sigma)}} C_{2}
$$

Its proof needs some preparation. Firstly, the numbers $\rho_{n, k}$ satisfy the following.

$$
\begin{aligned}
& \rho_{n, 0}= \begin{cases}1 & \text { for } n \geq 1, n \text { odd } \\
0 & \text { for } n \geq 0, n \text { even; }\end{cases} \\
& \rho_{n, k}=\rho_{n-1, k-1}+\rho_{n-1, k} \text { for } k, n \geq 1 \text {; } \\
& \rho_{n, k}=\sum_{i=1}^{n-1} \rho_{i, k-1} \quad \text { for } n \geq 0 \text { and } k \geq 1 \text {. }
\end{aligned}
$$

Equation (7.2) follows directly from the definition and equation (7.3) follows from an easy calculation. Then equation (7.4) follows by induction from equation (7.3).

Lemma 7.5. We have for $n \geq 1$ and $k \geq 0$

$$
H_{n}\left(C_{2}^{k} ; \mathbb{Z}\right) \cong \bigoplus_{i=1}^{t_{n, k}} C_{2}
$$

with $t_{n, k}=\sum_{j=1}^{k}\left(\begin{array}{c}k \\ j\end{array}\right) \cdot \rho_{n, j-1}$.

Proof. The assertion is obviously true for $k=0$. The induction step from $k-1$ to $k \geq 1$ is done as follows. Recall that $H_{n}\left(C_{2} ; \mathbb{Z}\right)$ is $\mathbb{Z}$ if $n=0, C_{2}$ if $n \geq 1$ and $n$ is odd, and $\{0\}$ otherwise. The Künneth formula gives the following short exact sequence of $\mathbb{Z}$-modules, which is natural in $C_{2}^{k-1}$

$$
\begin{aligned}
0 \rightarrow \bigoplus_{i+j=n} H_{i}\left(C_{2}^{k-1} ; \mathbb{Z}\right) \otimes_{\mathbb{Z}} H_{j}\left(C_{2} ; \mathbb{Z}\right) & \rightarrow H_{n}\left(C_{2}^{k-1} \times C_{2} ; \mathbb{Z}\right) \\
& \rightarrow \bigoplus_{i+j=n-1} \operatorname{Tor}_{1}^{\mathbb{Z}}\left(H_{i}\left(C_{2}^{k-1} ; \mathbb{Z}\right), H_{j}\left(C_{2} ; \mathbb{Z}\right)\right) \rightarrow 0
\end{aligned}
$$

It splits but the spitting is not natural in $C_{2}^{k-1}$. By rearranging the summands we obtain an isomorphism of $\mathbb{Z}$-modules

$$
H_{n}\left(C_{2}^{k} ; \mathbb{Z}\right) \cong H_{n}\left(C_{2} ; \mathbb{Z}\right) \oplus \bigoplus_{i=1}^{n} H_{i}\left(C_{2}^{k-1} ; \mathbb{Z}\right)
$$


Using the induction hypothesis we calculate

$$
\begin{aligned}
& t_{n, k}=t_{n, 1}+\sum_{i=1}^{n} t_{i, k-1} \\
& \stackrel{(7.2)}{=} \rho_{n, 0}+\sum_{i=1}^{n} \sum_{j=1}^{k-1}\left(\begin{array}{c}
k-1 \\
j
\end{array}\right) \cdot \rho_{i, j-1} \\
& =\rho_{n, 0}+\sum_{j=1}^{k-1}\left(\begin{array}{c}
k-1 \\
j
\end{array}\right) \cdot \sum_{i=1}^{n-1} \rho_{i, j-1}+\sum_{j=1}^{k-1}\left(\begin{array}{c}
k-1 \\
j
\end{array}\right) \cdot \rho_{n, j-1} \\
& \stackrel{(7.4)}{=} \rho_{n, 0}+\sum_{j=1}^{k-1}\left(\begin{array}{c}
k-1 \\
j
\end{array}\right) \cdot \rho_{n, j}+\sum_{j=1}^{k-1}\left(\begin{array}{c}
k-1 \\
j
\end{array}\right) \cdot \rho_{n, j-1} \\
& =\rho_{n, 0}+\sum_{j=2}^{k}\left(\begin{array}{c}
k-1 \\
j-1
\end{array}\right) \cdot \rho_{n, j-1}+\sum_{j=1}^{k-1}\left(\begin{array}{c}
k-1 \\
j
\end{array}\right) \cdot \rho_{n, j-1} \\
& =\sum_{j=1}^{k}\left(\begin{array}{c}
k-1 \\
j-1
\end{array}\right) \cdot \rho_{n, j-1}+\sum_{j=1}^{k}\left(\begin{array}{c}
k-1 \\
j
\end{array}\right) \cdot \rho_{n, j-1} \\
& =\sum_{j=1}^{k}\left(\left(\begin{array}{c}
k-1 \\
j-1
\end{array}\right)+\left(\begin{array}{c}
k-1 \\
j
\end{array}\right)\right) \cdot \rho_{n, j-1} \\
& =\sum_{j=1}^{k}\left(\begin{array}{l}
k \\
j
\end{array}\right) \cdot \rho_{n, j-1} \text {. }
\end{aligned}
$$

This finishes the proof of Lemma 7.5.

For $n \geq 1$ and $k \geq 0$ define

$$
S_{k} H_{n}:=\operatorname{cok}\left(\bigoplus_{i=1}^{k} H_{n}\left(j_{i}^{k} ; \mathbb{Z}\right): \bigoplus_{i=1}^{k} H_{n}\left(\left(C_{2}^{k}\right)_{i} ; \mathbb{Z}\right) \rightarrow H_{n}\left(C_{2}^{k} ; \mathbb{Z}\right)\right)
$$

Let the integer $s_{n, k} \geq 0$ be defined by

$$
S_{k} H_{n} \cong \bigoplus_{j=1}^{s_{n, k}} C_{2}
$$

Lemma 7.6. For $n \geq 1$ and $k \geq 1$ we have

$$
s_{n, k}=\rho_{n, k-1}
$$

and $s_{n, 0}=0$.

Proof. Since $H_{n}(\{1\} ; \mathbb{Z})=\{0\}$ for $n \geq 1$, we have $s_{n, 0}=0$. The induction step from $k-1$ to $k \geq 1$ is done as follows. Theorem 1.1 yields an isomorphism

$$
\bigoplus_{j=0}^{k} \bigoplus_{i=1}^{\left(\begin{array}{c}
k \\
j
\end{array}\right)} S_{j} H_{n} \cong H_{n}\left(C_{2}^{k} ; \mathbb{Z}\right)
$$


Using the induction hypothesis and Lemma 7.5 we conclude

$$
\begin{aligned}
s_{n, k} & =t_{n, k}-\sum_{j=0}^{k-1}\left(\begin{array}{l}
k \\
j
\end{array}\right) \cdot s_{n, j} \\
& =\sum_{j=1}^{k}\left(\begin{array}{l}
k \\
j
\end{array}\right) \rho_{n, j-1}-\sum_{j=1}^{k-1}\left(\begin{array}{l}
k \\
j
\end{array}\right) \cdot \rho_{n, j-1} \\
& =\rho_{n, k-1} .
\end{aligned}
$$

This finishes the proof of Lemma 7.6.

Now Theorem 7.1 follows from Theorem 1.1 applied to the equivariant homology theory given by Borel homology and singular homology with $\mathbb{Z}$-coefficients, see Example 2.3, and from Lemma 7.6. Here we use the fact that for any group $G$ the space $E G \times{ }_{G} \underline{E} G$ is a model for $B G$.

Remark 7.7. If we replace in this subsection $C_{2}=\mathbb{Z} / 2$ everywhere by $\mathbb{Z} / p^{l}$ for some prime number $p$ and some natural number $l$, then Theorem 7.1 remains true. This follows from two facts. Since $\mathbb{Z} / p^{l}$ is a local ring, we conclude from [29, Lemma 1.2 on page 5] that for every natural number $a$, every summand of the abelian group $\bigoplus_{i=1}^{a} \mathbb{Z} / p^{l}$ is isomorphic to $\bigoplus_{j=1}^{b} \mathbb{Z} / p^{l}$ for some natural number $b$. The group homology $H_{n}\left(\mathbb{Z} / p^{l} ; \mathbb{Z}\right)$ is isomorphic to $\mathbb{Z} / p^{l}$ if $n$ is odd and vanishes for even $n$ with $n \geq 2$.

\subsection{Negative $K$-groups for $R=\mathbb{Z}$.}

Theorem 7.8. We have $K_{n}(\mathbb{Z} W)=\{0\}$ for $n \leq-1$.

Proof. Since right-angled Coxeter groups satisfy the Farrell-Jones Conjecture, we get $K_{n}(\mathbb{Z} W)=0$ for $n \leq-2$ and an isomorphism

$$
\operatorname{colim}_{H \in \operatorname{Sub}_{\mathcal{F I N}}(W)} K_{-1}(\mathbb{Z} H) \rightarrow K_{-1}(\mathbb{Z} W)
$$

from [27, page 749].

Since any finite subgroup of $W$ is isomorphic to $(\mathbb{Z} / 2)^{k}$ for some natural number $k$, and $K_{-1}(\mathbb{Z} A)=0$ holds for a finite abelian group whose order is a prime power, see [4, Theorem 10.6 on page 695 ] or [8], the claim follows.

7.3. Projective class group for $R=\mathbb{Z}$.

\section{Theorem 7.9.}

(i) There is an isomorphism

$$
K_{0}(\mathbb{Z}) \oplus \bigoplus_{\sigma \in \mathcal{P}} \mathbb{Z} /\left(2^{\operatorname{dim} \sigma-1}\right) \stackrel{\cong}{\longrightarrow} H_{0}^{W}\left(\underline{E} W ; \mathbf{K}_{\mathbb{Z}}\right) ;
$$

(ii) The map

$$
H_{0}^{W}\left(\underline{E} W ; \mathbf{K}_{\mathbb{Z}}\right) \rightarrow H_{0}^{W}\left(\underline{\underline{E}} W ; \mathbf{K}_{\mathbb{Z}}\right)
$$

is an isomorphism after inverting 2 ;

(iii) The canonical map

$$
H_{0}^{W}\left(\underline{\underline{E}} W ; \mathbf{K}_{\mathbb{Z}}\right) \rightarrow K_{0}(\mathbb{Z} W)
$$

is an isomorphism;

(iv) We have $\widetilde{K}_{0}(\mathbb{Z} W) \otimes_{\mathbb{Z}} \mathbb{Z}[1 / 2]=\{0\}$.

Proof. (i) We have for every group $G$ the obvious splitting $K_{0}(\mathbb{Z} G) \cong K_{0}(\mathbb{Z}) \oplus$ $\widetilde{K}_{0}(\mathbb{Z} G)$. By [39, Theorem 12.9], $\widetilde{K}_{0}\left(\mathbb{Z}\left[C_{2}^{k}\right]\right) \cong \bigoplus_{i=3}^{k}\left(\begin{array}{c}k \\ i\end{array}\right) \mathbb{Z} /\left(2^{i-2}\right)$. This implies that for $H_{*}^{\text {? }}\left(-; \mathbf{K}_{\mathbb{Z}}\right)$ the groups $S_{\sigma}$ in Theorem 1.1 are given by $\mathbb{Z} /\left(2^{\operatorname{dim} \sigma-1}\right)$. Now the assertion follows from Theorem 1.1 applied to the equivariant homology theory $H_{*}^{?}\left(-; \mathbf{K}_{\mathbb{Z}}\right)$. 
(ii) This follows from Theorem 5.3 (iv).

(iii) This follows from the fact that a right-angled Coxeter group satisfies the Farrell--Jones Conjecture.

(iv) This follows from assertions (i), (ii), and (iii).

\subsection{Whitehead group.}

\section{Theorem 7.10.}

(i) The canonical map

$$
H_{1}^{W}\left(E W ; \mathbf{K}_{\mathbb{Z}}\right) \rightarrow H_{1}^{W}\left(\underline{E} W ; \mathbf{K}_{\mathbb{Z}}\right)
$$

is an isomorphism and we have an isomorphism

$$
H_{1}^{W}\left(E W ; \mathbf{K}_{\mathbb{Z}}\right) \cong H_{1}(W ; \mathbb{Z}) \oplus K_{1}(\mathbb{Z}) ;
$$

(ii) The map

$$
H_{1}^{W}\left(\underline{E} W ; \mathbf{K}_{\mathbb{Z}}\right) \rightarrow H_{1}^{W}\left(\underline{\underline{E}} W ; \mathbf{K}_{\mathbb{Z}}\right)
$$

is an isomorphism after inverting 2 ;

(iii) The canonical map

$$
H_{1}^{W}\left(\underline{\underline{E}} W ; \mathbf{K}_{\mathbb{Z}}\right) \rightarrow K_{1}(\mathbb{Z} W)
$$

is an isomorphism;

(iv) We have $K_{1}(\mathbb{Z} W) \otimes_{\mathbb{Z}} \mathbb{Z}[1 / 2]=\{0\}$.

Proof. (i) Notice that we have isomorphisms

$$
\begin{aligned}
H_{1}^{W}\left(E W ; \mathbf{K}_{\mathbb{Z}}\right) \cong H_{1}(B W ; \mathbf{K}(\mathbb{Z})) & \cong H_{1}\left(B W, K_{0}(\mathbb{Z})\right) \oplus H_{0}\left(B W, K_{1}(\mathbb{Z})\right) \\
& \cong H_{1}(W ; \mathbb{Z}) \oplus K_{1}(\mathbb{Z}) \cong W /[W, W] \oplus\{ \pm 1\} .
\end{aligned}
$$

Hence it remains to show that the canonical map $H_{1}^{W}\left(E W ; \mathbf{K}_{\mathbb{Z}}\right) \rightarrow H_{1}^{W}\left(\underline{E} W ; \mathbf{K}_{\mathbb{Z}}\right)$ is bijective. The Whitehead group $\mathrm{Wh}\left(C_{2}^{k}\right)$ vanishes for all natural numbers $k$ by [30, Theorem 14.2 (iii) on page 330]. Hence the obvious map $H_{1}\left(C_{2}^{k} ; \mathbb{Z}\right) \times$ $K_{1}(\mathbb{Z}) \rightarrow K_{1}\left(\mathbb{Z}\left[C_{2}^{k}\right]\right)$ is an isomorphism. Now apply Theorem 1.1 to the equivariant homology theories given by the Borel construction, see Example 2.3, and to $H_{*}^{?}\left(-; \mathbf{K}_{\mathbb{Z}}\right)$.

(ii) This follows from Theorem 5.3 (iv).

(iii) This follows from the fact that a right-angled Coxeter group satisfies the Farrell--Jones Conjecture.

(iv) This follows from Theorem 7.1 and assertions (i), (ii), and (iii).

7.5. Rationalized $K$-groups. Let $R$ be a ring. For any non-empty simplex $\sigma$ of $\Sigma$ we have the diagonal embedding

$$
\Delta_{\sigma}: C_{2} \rightarrow W(\sigma)=\prod_{v \in V \cap \sigma} W_{v}=\prod_{v \in V \cap \sigma} C_{2} .
$$

Let $j_{\sigma}: W(\sigma) \rightarrow W$ be the inclusion. Then $j_{\sigma} \circ \Delta_{\sigma}: C_{2} \rightarrow W$ induces a homomorphisms $\left(j_{\sigma} \circ \Delta_{\sigma}\right)_{*}: K_{n}\left(R\left[C_{2}\right]\right) \rightarrow K_{n}(R W)$. Denote by

$$
i_{\sigma, n}: \operatorname{ker}\left(K_{n}\left(R\left[C_{2}\right]\right) \rightarrow K_{n}(R)\right) \rightarrow K_{n}(R W)
$$

its composite with the inclusion $\operatorname{ker}\left(K_{n}\left(R\left[C_{2}\right]\right) \rightarrow K_{n}(R)\right) \rightarrow K_{n}\left(R\left[C_{2}\right]\right)$, where $K_{n}\left(R\left[C_{2}\right]\right) \rightarrow K_{n}(R)$ is the homomorphism induced by the projection $C_{2} \rightarrow\{1\}$. Let $i_{\emptyset, n}: K_{n}(R) \rightarrow K_{n}(R W)$ be the map induced by the inclusion $\{1\} \rightarrow W$.

Theorem 7.11. Let $R$ be a regular ring. 
(i) The map

$$
i_{\emptyset, n} \oplus \bigoplus_{\substack{\sigma \in \mathcal{P} \\ \sigma \neq \emptyset}} i_{\sigma, n}: K_{n}(R) \oplus \bigoplus_{\substack{\sigma \in \mathcal{P} \\ \sigma \neq \emptyset}} \operatorname{ker}\left(K_{n}\left(R\left[C_{2}\right]\right) \rightarrow K_{n}(R)\right) \rightarrow K_{n}(R W)
$$

is rationally an isomorphism for all $n \in \mathbb{Z}$;

(ii) We have for $R=\mathbb{Z}$

$$
\mathbb{Q} \otimes_{\mathbb{Z}} K_{n}(\mathbb{Z} W) \cong \begin{cases}\mathbb{Q}^{r} & \text { if } n=4 k+1 \text { with } k \geq 1 ; \\ \mathbb{Q} & \text { if } n=0 ; \\ \{0\} & \text { otherwise. }\end{cases}
$$

Proof. (i) Notice that any non-trivial finite cyclic subgroup $C$ of $C_{2}^{k}$ is isomorphic to $C_{2}$ and that the obvious composite

$$
\begin{aligned}
\operatorname{ker}\left(\mathbb{Q} \otimes_{\mathbb{Z}} K_{n}(R C) \rightarrow \mathbb{Q} \otimes_{\mathbb{Z}} K_{n}(R)\right) \rightarrow & \mathbb{Q} \otimes_{\mathbb{Z}} K_{n}(R C) \\
& \rightarrow \operatorname{cok}\left(\mathbb{Q} \otimes_{\mathbb{Z}} K_{n}(R) \rightarrow \mathbb{Q} \otimes_{\mathbb{Z}} K_{n}(R C)\right)
\end{aligned}
$$

is an isomorphism. The isomorphism appearing in $[1,(2.11)]$, which exists for $\Lambda=\mathbb{Q}$ and the equivariant homology theory $H_{*}^{?}\left(-; \mathbf{K}_{R}\right)$ because of $[1$, Lemma 4.1 (e)], which in turn follows from [36, Corollary 4.2], boils down to an isomorphism

$$
\begin{array}{r}
j_{\{1\}} \oplus \bigoplus_{\substack{C \subseteq C_{2}^{k} \\
C \cong C_{2}}} j_{C}: \mathbb{Q} \otimes_{\mathbb{Z}} K_{n}(R) \oplus \underset{\substack{C \subseteq C_{2}^{k} \\
C \cong C_{2}}}{\bigoplus} \operatorname{ker}\left(\varepsilon_{C}^{\mathbb{Q}}: \mathbb{Q} \otimes_{\mathbb{Z}} K_{n}(R C) \rightarrow \mathbb{Q} \otimes_{\mathbb{Z}} K_{n}(R)\right) \\
\\
\stackrel{\cong}{\longrightarrow} \otimes_{\mathbb{Z}} K_{n}\left(R\left[C_{2}^{k}\right]\right),
\end{array}
$$

where $\varepsilon_{C}^{\mathbb{Q}}: \mathbb{Q} \otimes_{\mathbb{Z}} K_{n}(R C) \rightarrow \mathbb{Q} \otimes_{\mathbb{Z}} K_{n}(R)$ is induced by the projection $C \rightarrow\{1\}$, the map $j_{\{1\}}$ is induced by the inclusion $\{1\} \rightarrow C$, and $j_{C}$ is the composite of the inclusion $\operatorname{ker}\left(\varepsilon_{C}^{\mathbb{Q}}\right) \rightarrow \mathbb{Q} \otimes_{\mathbb{Z}} K_{n}(R C)$ with the map $\mathbb{Q} \otimes_{\mathbb{Z}} K_{n}(R C) \rightarrow \mathbb{Q} \otimes_{\mathbb{Z}} K_{n}\left(R\left[C_{2}^{k}\right]\right)$ coming from the inclusion $C \rightarrow C_{2}^{k}$. By naturality we get a commutative diagram

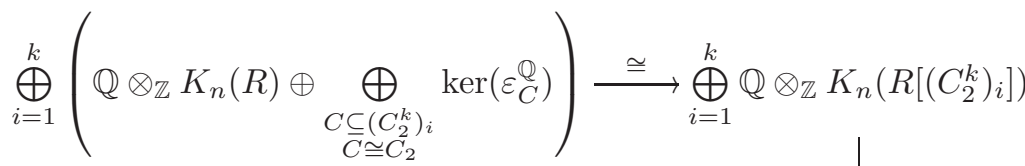

$$
\begin{aligned}
& \downarrow \\
& \mathbb{Q} \otimes_{\mathbb{Z}} K_{n}(R) \oplus \underset{\substack{C \subseteq C_{2}^{k} \\
C \cong C_{2}}}{\bigoplus} \operatorname{ker}\left(\varepsilon_{C}^{\mathbb{Q}}\right) \longrightarrow \mathbb{Q} \otimes_{\mathbb{Z}} K_{n}\left(R\left[C_{2}^{k}\right]\right)
\end{aligned}
$$

where the vertical arrows come from the inclusions $\left(C_{2}^{k}\right)_{i} \rightarrow C_{2}^{k}$. Notice that a cyclic subgroup of $C_{2}^{k}$ belongs to $\left(C_{2}^{k}\right)_{i}$ for some $i \in\{1,2, \ldots, k\}$ if and only if it is different from the diagonal subgroup $\left(C_{2}^{k}\right)_{\Delta}:=\left\{(a, a, \ldots, a) \mid a \in C_{2}\right\} \subseteq C_{2}^{k}$. Hence the composite

$$
\begin{aligned}
& \operatorname{ker}\left(K_{n}\left(R\left[\left(C_{2}^{k}\right)_{\Delta}\right]\right) \rightarrow K_{n}(R)\right) \rightarrow K_{n}\left(R\left[\left(C_{2}^{k}\right)_{\Delta}\right]\right) \rightarrow K_{n}\left(R\left[C_{2}^{k}\right]\right) \\
& \rightarrow \operatorname{cok}\left(\bigoplus_{i=1}^{k} K_{n}\left(R\left[\left(C_{2}^{k}\right)_{i}\right]\right) \rightarrow K_{n}\left(R\left[C_{2}^{k}\right]\right)\right)
\end{aligned}
$$

is rationally bijective.

Now assertion (i) follows from Theorem 1.1 and Theorem 5.3 (iv). 
(ii) Due to Borel [5] we know for $R=\mathbb{Z}$ that

$$
\mathbb{Q} \otimes_{\mathbb{Z}} K_{n}(\mathbb{Z}) \cong \begin{cases}\mathbb{Q} & \text { if } n=4 k+1 \text { with } k \geq 1 ; \\ \mathbb{Q} & \text { if } n=0 ; \\ \{0\} & \text { otherwise. }\end{cases}
$$

We get from [20, Theorem 2.2] for $C \cong C_{2}$

$$
\mathbb{Q} \otimes_{\mathbb{Z}} K_{n}(\mathbb{Z}[C]) \cong \begin{cases}\mathbb{Q}^{2} & \text { if } n=4 k+1 \text { with } k \geq 1 ; \\ \mathbb{Q} & \text { if } n=0 ; \\ \{0\} & \text { otherwise. }\end{cases}
$$

Hence we get

$$
\operatorname{ker}\left(\mathbb{Q} \otimes_{\mathbb{Z}} K_{n}(\mathbb{Z} C) \rightarrow \mathbb{Q} \otimes_{\mathbb{Z}} K_{n}(\mathbb{Z})\right) \cong \begin{cases}\mathbb{Q} & \text { if } n=4 k+1 \text { with } k \geq 1 \\ \{0\} & \text { otherwise. }\end{cases}
$$

Now assertion (ii) follows from assertion (i).

7.6. L-groups after inverting 2. The maps appearing in the result below are defined analogously to the maps appearing in Theorem 7.11.

Theorem 7.12. Let $R$ be a ring with involution.

(i) The map

$$
\begin{gathered}
i_{\emptyset, n} \oplus \bigoplus_{\substack{\sigma \in \mathcal{P} \\
\sigma \neq \emptyset}} i_{\sigma, n}: L_{n}^{\langle-\infty\rangle}(R) \oplus \bigoplus_{\substack{\sigma \in \mathcal{P} \\
\sigma \neq \emptyset}} \operatorname{ker}\left(L_{n}^{\langle-\infty\rangle}\left(R\left[C_{2}\right]\right) \rightarrow L_{n}^{\langle-\infty\rangle}(R)\right) \rightarrow L_{n}^{\langle-\infty\rangle}(R W) \\
\quad \text { is an isomorphism after inverting } 2 \text { for all } n \in \mathbb{Z} ;
\end{gathered}
$$

(ii) We have for $R \in\{\mathbb{Z}, \mathbb{Q}, \mathbb{R}\}$

$$
L_{n}^{\langle-\infty\rangle}(R W)[1 / 2] \cong \begin{cases}\mathbb{Z}[1 / 2]^{r} & \text { if } n=4 k \text { for } k \in \mathbb{Z} \\ \{0\} & \text { otherwise }\end{cases}
$$

Proof. (i) Note that any non-trivial subgroup of the form $C \times P$ of $C_{2}^{k}$ for a cyclic group $C$ and a $p$-group $P$ for an odd prime number $p$ is isomorphic to $C_{2}$. The isomorphism appearing in $[1,(2.11)]$ which exists for $\Lambda=\mathbb{Z}[1 / 2]$ and the equivariant homology theory $H_{*}^{?}\left(-; \mathbf{L}_{R}^{\langle-\infty\rangle}\right)$ because of $[14$, Theorem 2], boils down to an isomorphism

$$
\begin{aligned}
& j_{\{1\}} \oplus \bigoplus_{\substack{C \subseteq C_{2}^{k} \\
C \cong C_{2}}} j_{C}: L_{n}^{\langle-\infty\rangle}(R)[1 / 2] \oplus \bigoplus_{\substack{C \subseteq C_{2}^{k} \\
C \cong C_{2}}} \operatorname{ker}\left(L_{n}^{\langle-\infty\rangle}(R C)[1 / 2] \rightarrow L_{n}^{\langle-\infty\rangle}(R)[1 / 2]\right) \\
& \stackrel{\cong}{\longrightarrow} L_{n}^{\langle-\infty\rangle}\left(R\left[C_{2}^{k}\right]\right)[1 / 2],
\end{aligned}
$$

where the map $j_{\{1\}}$ is induced by the inclusion $\{1\} \rightarrow C$, and $j_{C}$ is the composite of the inclusion of $\operatorname{ker}\left(L_{n}^{\langle-\infty\rangle}(R C)[1 / 2] \rightarrow L_{n}^{\langle-\infty\rangle}(R)[1 / 2]\right)$ into $L_{n}^{\langle-\infty\rangle}(R C)[1 / 2]$ with the map $L_{n}^{\langle-\infty\rangle}(R C)[1 / 2] \rightarrow L_{n}^{\langle-\infty\rangle}\left(R\left[C_{2}^{k}\right]\right)[1 / 2]$ coming from the inclusion $C \rightarrow$ $C_{2}^{k}$. Now Theorem 7.12 follows completely analogous to the argument appearing in the proof of Theorem 7.11 (i).

(ii) This follows from assertion (i) using [33, Proposition 22.34 on page 254].

\section{7. $K$ - and $L$-groups for $R$ containing $1 / 2$.}

Theorem 7.13. For all $n \in \mathbb{Z}$ there are explicit isomorphisms

(i) $\bigoplus_{\sigma \in \mathcal{P}} K_{n}(R) \cong K_{n}(R W)$ if $R$ is regular and contains $1 / 2$;

(ii) $\bigoplus_{\sigma \in \mathcal{P}} L_{n}^{\langle-\infty\rangle}(R) \stackrel{\cong}{\longrightarrow} L_{n}^{\langle-\infty\rangle}(R W)$ if $R$ contains $1 / 2$. 
Its proof needs some preparations. In the sequel we will write $C_{2}^{k}$ multiplicatively and we denote by $t_{i}$ the generator of the $i$-th factor $C_{2}$ viewed as an element in $C_{2}^{k}$ for $i=1,2, \ldots, k$.

Let $R$ be a ring in which 2 is invertible. We get a decomposition of rings, natural in $R$,

$$
R\left[C_{2}\right] \stackrel{\cong}{\longrightarrow} R \times R, \quad a+b t \mapsto(a+b, a-b) .
$$

Its inverse sends $(c, d)$ to $\frac{1}{2} \cdot((c+d)+(c-d) \cdot t)$. Since algebraic $K$-theory is compatible with products, we obtain an isomorphism, natural in $R$,

$$
S_{n}(R): K_{n}\left(R\left[C_{2}\right]\right) \stackrel{\cong}{\rightrightarrows} K_{n}(R) \times K_{n}(R) .
$$

One can iterate this using the obvious ring isomorphism $\left(R\left[C_{2}^{k-1}\right]\right)\left[C_{2}\right] \cong R\left[C_{2}^{k}\right]$ and thus obtains an isomorphism

$$
S_{n}^{k}(R): K_{n}\left(R\left[C_{2}^{k}\right]\right) \stackrel{\cong}{\rightarrow} \prod_{\epsilon \in \operatorname{hom}_{\mathbb{Z}}\left(C_{2}^{k},\{ \pm 1\}\right)} K_{n}(R)
$$

which comes from the isomorphism of rings

$$
R\left[C_{2}^{k}\right] \stackrel{\rightrightarrows}{\rightarrow} \prod_{\epsilon \in \operatorname{hom}_{\mathbb{Z}}\left(C_{2}^{k},\{ \pm 1\}\right)} R, \quad \sum_{g \in C_{2}^{k}} \lambda_{g} \cdot g \mapsto\left(\sum_{g \in C_{2}^{k}} \lambda_{g} \cdot \epsilon(g)\right)_{\epsilon} .
$$

Its inverse is given by

$$
\left(\mu_{\epsilon}\right)_{\epsilon} \mapsto 2^{-k} \cdot \sum_{g \in C_{2}^{k}}\left(\sum_{\epsilon} \epsilon(g) \cdot \mu_{\epsilon}\right) \cdot g
$$

Lemma 7.15. (i) Suppose that 2 is invertible in $R$. Then there is an isomorphism

$$
d_{n}^{k}: \operatorname{cok}\left(\bigoplus_{i=1}^{k} K_{n}\left(R\left[j_{i}^{k}\right]\right): \bigoplus_{i=1}^{k} K_{n}\left(R\left[\left(C_{2}^{k}\right)_{i}\right]\right) \rightarrow K_{n}\left(R\left[C_{2}^{k}\right]\right)\right) \cong K_{n}(R) .
$$

Its inverse is the composite of the homomorphism

$$
\beta: K_{n}(R) \rightarrow K_{n}\left(R\left[C_{2}^{k}\right]\right)
$$

coming from the ring homomorphism $R \rightarrow R\left[C_{2}^{k}\right]$ sending $\lambda$ to $2^{-k} \cdot \lambda$. $\prod_{i=1}^{k}\left(1-t_{i}\right)$ with the projection $K_{n}\left(R\left[C_{2}^{k}\right]\right) \rightarrow \operatorname{cok}\left(\bigoplus_{i=1}^{k} K_{n}\left(R\left[j_{i}^{k}\right]\right)\right)$. The homomorphism $\beta$ agrees with the restriction of the inverse of the isomorphism $S_{n}^{k}(R)$ of (7.14) to the factor $K_{n}(R)$ which belongs to $\epsilon$ given by $\epsilon\left(t_{i}\right)=-1$ for $i=1,2, \ldots k$;

(ii) The same assertion holds if we replace algebraic $K$-theory by algebraic $L$-theory with the decoration $\langle-\infty\rangle$;

(iii) The same assertion is true if we take $R$ to be $\mathbb{R}$ or $\mathbb{C}$ and we replace algebraic $K$-theory by topological $K$-theory.

Proof. We give the proof for algebraic $K$-theory only, the one for the other cases is completely analogous.

We use induction over $k$. If $k=0$, the map $d_{n}^{0}$ comes from the identification

$$
K_{n}\left(R\left[C_{2}^{0}\right]\right)=K_{n}(R[\{1\}])=K_{n}(R) .
$$


The induction step from $(k-1)$ to $k \geq 1$ is done as follows. We have the following commutative diagram of $\mathbb{Z}$-modules

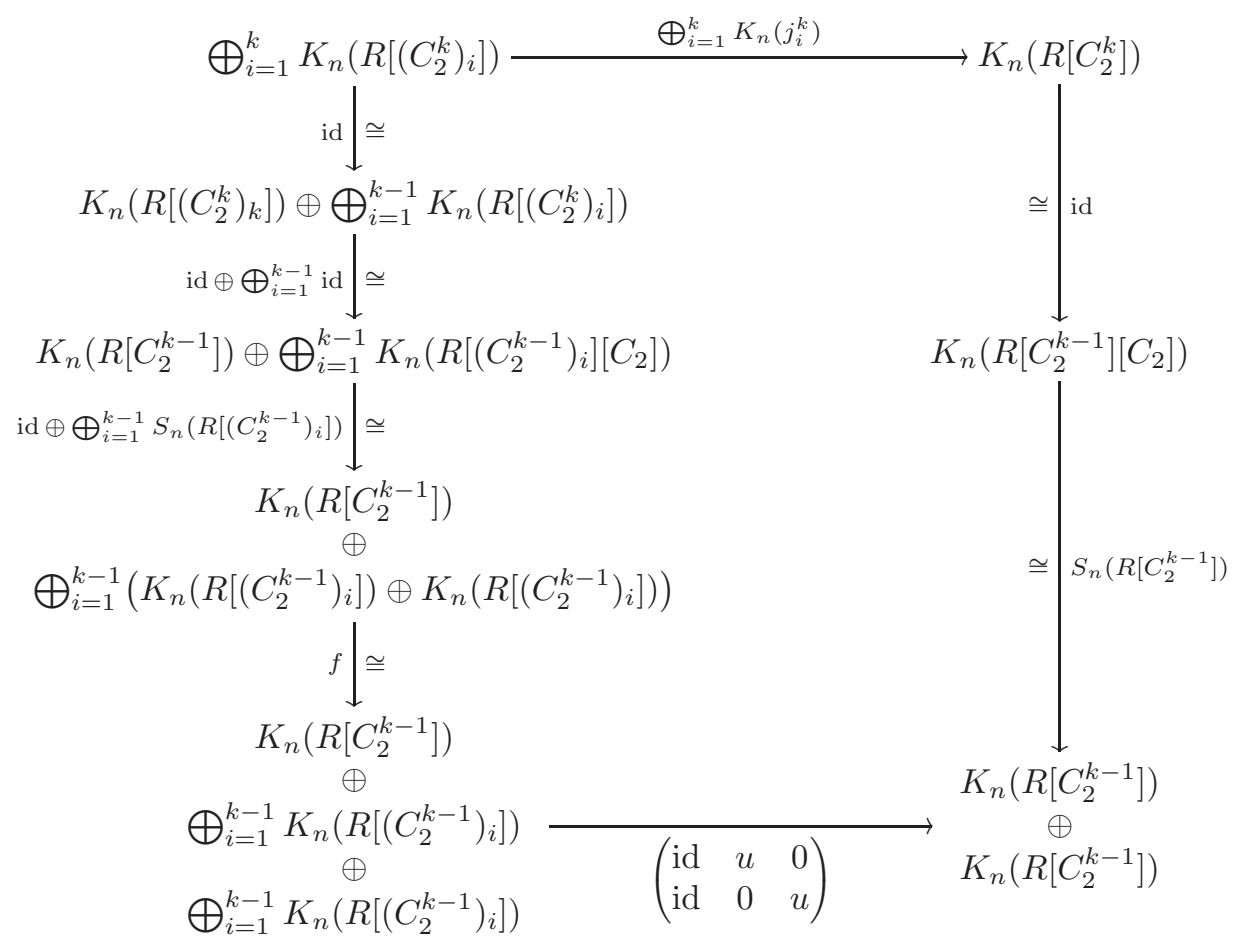

where $f$ is the obvious isomorphism and $u:=\bigoplus_{i=1}^{k-1} K_{n}\left(j_{i}^{k-1}\right)$. The diagram above induces an isomorphism

$$
\operatorname{cok}\left(\bigoplus_{i=1}^{k} K_{n}\left(j_{i}^{k}\right)\right) \stackrel{\cong}{\longrightarrow} \operatorname{cok}\left(\begin{array}{ccc}
\text { id } & u & 0 \\
\text { id } & 0 & u
\end{array}\right)
$$

If $k=1$, then $\left(\begin{array}{ccc}\text { id } & u & 0 \\ \text { id } & 0 & u\end{array}\right)$ reduces to $K_{n}(R) \rightarrow K_{n}(R) \oplus K_{n}(R), x \mapsto(x, x)$ and the desired isomorphism $d_{n}^{1}$ is induced by $K_{n}(R) \oplus K_{n}(R) \rightarrow K_{n}(R),(x, y) \mapsto x-y$. Suppose $k \geq 2$. Since the first and third map in the composite

$$
\left(\begin{array}{cc}
\mathrm{id} & 0 \\
-\mathrm{id} & \mathrm{id}
\end{array}\right) \cdot\left(\begin{array}{ccc}
\mathrm{id} & u & 0 \\
\mathrm{id} & 0 & u
\end{array}\right) \cdot\left(\begin{array}{ccc}
\mathrm{id} & 0 & 0 \\
0 & \mathrm{id} & 0 \\
0 & \mathrm{id} & \mathrm{id}
\end{array}\right)
$$

are isomorphisms and the composite is given by $\left(\begin{array}{ccc}\text { id } & u & 0 \\ 0 & 0 & u\end{array}\right)$, we obtain an isomorphism

$$
\operatorname{cok}\left(\begin{array}{ccc}
\text { id } & u & 0 \\
\text { id } & 0 & u
\end{array}\right) \cong \operatorname{cok}\left(\begin{array}{ccc}
\text { id } & u & 0 \\
0 & 0 & u
\end{array}\right) \text {. }
$$

Since (id $u): K_{n}\left(R\left[C_{2}^{k-1}\right]\right) \oplus \bigoplus_{i=1}^{k-1} K_{n}\left(R\left[\left(C_{2}^{k-1}\right)_{i}\right]\right) \rightarrow K_{n}\left(R\left[C_{2}^{k-1}\right]\right)$ is surjective, we obtain an isomorphism

$$
\operatorname{cok}\left(\begin{array}{ccc}
\text { id } & u & 0 \\
0 & 0 & u
\end{array}\right) \stackrel{\cong}{\longrightarrow} \operatorname{cok}(u) .
$$

Its inverse is induced by the composite

$$
K_{n}\left(R\left[C_{2}^{k-1}\right]\right) \stackrel{\left(\begin{array}{c}
0 \\
\mathrm{id}
\end{array}\right)}{\longrightarrow} K_{n}\left(R\left[C_{2}^{k-1}\right]\right) \oplus K_{n}\left(R\left[C_{2}^{k-1}\right]\right) \stackrel{S_{n}\left(R\left[C_{2}^{k-1}\right]\right)^{-1}}{\longrightarrow} K_{n}\left(R\left[C_{2}^{k}\right]\right),
$$


which is the homomorphism $K_{n}\left(R\left[C_{2}^{k-1}\right]\right) \rightarrow K_{n}\left(R\left[C_{2}^{k}\right]\right)$ induced by the ring homomorphism $R\left[C_{2}^{k-1}\right] \rightarrow R\left[C_{2}^{k}\right]$ sending $x$ to $\frac{1}{2} \cdot x \cdot\left(1-t_{k}\right)$. Since the induction hypothesis applies to $u$, Lemma 7.15 follows.

Now Theorem 7.13 follows from Theorem 1.1, Theorem 5.3 (iii) and (vi), and Lemma 7.15.

\subsection{Topological $K$-theory.}

Theorem 7.16. There are for every $n \in \mathbb{Z}$ isomorphisms

$$
\begin{aligned}
\bigoplus_{\sigma \in \mathcal{P}} K_{n}(\mathbb{C}) & \cong \\
\bigoplus_{\sigma \in \mathcal{P}} K O_{n}(\mathbb{R}) & \left.\cong C_{m}^{*}(W)\right) \cong K_{n}\left(C_{r}^{*}(W)\right) ;
\end{aligned}
$$

In particular there are isomorphisms of abelian groups

$$
\begin{array}{r}
K_{n}\left(C_{m}^{*}(W)\right) \cong K_{n}\left(C_{r}^{*}(W)\right) \cong \begin{cases}\mathbb{Z}^{r} \text { if } n \text { is even } ; \\
\{0\} \quad \text { otherwise } ;\end{cases} \\
K O_{n}\left(C_{m}^{*}(W ; \mathbb{R})\right) \cong K O_{n}\left(C_{r}^{*}(W ; \mathbb{R})\right) \cong \begin{cases}\mathbb{Z}^{r} & \text { if } n \equiv 0 \bmod 4 ; \\
(\mathbb{Z} / 2)^{r} & \text { if } n \equiv 1,2 \bmod 8 ; \\
\{0\} & \text { otherwise. }\end{cases}
\end{array}
$$

Proof. This follows from Theorem 1.1 and Lemma 7.15.

The result for complex coefficients was already obtained by Sánchez-García using the Davis complex as a model for $\underline{E} W$ in [34]. In special cases, the topological $K$ theory of $\underline{E} W$ was computed in Fuentes Rumís masters's thesis [16].

Remark 7.17. In Subsection 1.3 we have given an explicit description of the isomorphism $\bigoplus_{\sigma \in \mathcal{P}} K_{n}(\mathbb{C}) \stackrel{\cong}{\rightrightarrows} K_{n}\left(C_{r}^{*}(G)\right)$ above which actually carries over to many of the other situations. In order to prove the description, one has to go through the construction of the isomorphism and to make in the application of Lemma 4.5 the right choice for $\widehat{s}_{\tau}$. Namely, one takes for $\widehat{s}_{\tau}$ the composite of the homomorphism $\beta$ with the isomorphism $d_{n}^{k}$ appearing in assertion (i) of Lemma 7.15.

\section{An example}

In this section, we want to apply the computations from the previous sections to a concrete example. For this we picked the group $W:=\mathbb{Z} / 2 \times \mathbb{Z} / 2 \times D_{\infty} \times \mathbb{Z}$. Note that it is a graph product with vertex groups $\mathbb{Z} / 2$ and $\mathbb{Z}$. In [10, Example 3.28] Davis, Khan and Ranicki showed that the Whitehead group of $W$ is infinitely generated due to Nil elements.

It will be useful to consider $W$ as $W_{0} \times \mathbb{Z}$, where $W_{0}=\mathbb{Z} / 2 \times \mathbb{Z} / 2 \times D_{\infty}$ is the right-angled Coxeter group associated to the simplicial graph $X$ with vertex set $V=\{1,2,3,4\}$ whose edges are $\{1,2\},\{2,3\},\{3,4\},\{1,4\}$, and $\{1,3\}$. Then the flag complex $\Sigma$ associated to $X$ is the suspension of a one-simplex so that in the notation of Section 7 we have $r_{-1}=1, r_{0}=4, r_{1}=5, r_{2}=2$, and $r=12$.

We conclude from Theorem 7.1 for $n \geq 2$

$$
\begin{aligned}
& H_{n}(W ; \mathbb{Z}) \cong H_{n}\left(W_{0} ; \mathbb{Z}\right) \oplus H_{n-1}\left(W_{0} ; \mathbb{Z}\right) \cong \bigoplus_{i=1}^{u_{n}} C_{2} ; \\
& H_{1}(W ; \mathbb{Z}) \cong \mathbb{Z} \oplus \bigoplus_{i=1}^{4} C_{2},
\end{aligned}
$$


where

$$
\begin{aligned}
u_{n} & =\sum_{k=0}^{2} r_{k} \cdot \rho_{n, k}+\sum_{k=0}^{2} r_{k} \cdot \rho_{n-1, k} \\
& =4 \cdot\left(\rho_{n, 0}+\rho_{n-1,0}\right)+5 \cdot\left(\rho_{n, 1}+\rho_{n-1,1}\right)+2 \cdot\left(\rho_{n, 2}+\rho_{n-1,2}\right) \\
& = \begin{cases}4+5(k+k-1)+2\left(k(k-1)+(k-1)^{2}\right) & \text { if } n=2 k \text { for } k \geq 1 ; \\
4+5(k+k)+2\left(k^{2}+k(k-1)\right) & \text { if } n=2 k+1 \text { for } k \geq 1 ;\end{cases} \\
& = \begin{cases}4 k^{2}+4 k+1 & \text { if } n=2 k \text { for } k \geq 1 ; \\
4 k^{2}+8 k+4 & \text { if } n=2 k+1 \text { for } k \geq 1 .\end{cases}
\end{aligned}
$$

Note that the group $W$ satisfies the Baum-Connes Conjecture and the FarrellJones Conjecture since it is a graph product of abelian groups. Hence for every regular ring $R$ the assembly map

$$
H_{n}^{W}\left(\underline{E} W ; \mathbf{K}_{R}\right) \rightarrow K_{n}(R[W])
$$

is bijective after inverting 2 by Theorem 5.3 (iv).

The proof of Theorem 7.8 applies verbatim to the group $W$ so that we obtain

$$
K_{n}(\mathbb{Z} W)=\{0\} \quad \text { for } n \leq-1 .
$$

For any equivariant homology theory we have

$$
\mathcal{H}_{n}^{W}(\underline{E} W) \cong \mathcal{H}_{n}^{W_{0}}\left(\underline{E} W_{0} \times S^{1}\right) \cong \mathcal{H}_{n}^{W_{0}}\left(\underline{E} W_{0}\right) \oplus \mathcal{H}_{n-1}^{W_{0}}\left(\underline{E} W_{0}\right) .
$$

Using (8.1), we have

$$
\mathbb{Z}[1 / 2] \otimes_{\mathbb{Z}} \widetilde{K}_{0}(\mathbb{Z} W)=\{0\}
$$

by Theorem 7.9 and Theorem 7.8 .

By (8.1), Theorem 7.9 and Theorem 7.10, we have

$$
\mathbb{Z}[1 / 2] \otimes_{\mathbb{Z}} K_{1}(\mathbb{Z} W) \cong \mathbb{Z}[1 / 2] \quad \text { and } \quad \mathbb{Z}[1 / 2] \otimes_{\mathbb{Z}} \mathrm{Wh}(W)=\{0\} .
$$

Note that without inverting two, the Whitehead group $\mathrm{Wh}(W)$ contains a nontrivial Nil term by [10, Example 3.28] as mentioned above.

By (8.1) and Theorem 7.11

$$
\mathbb{Q} \otimes_{\mathbb{Z}} K_{n}(\mathbb{Z} W) \cong \begin{cases}\mathbb{Q}^{12} & \text { if } n=4 k+1 \text { or } n=4 k+2 \text { for } k \geq 1 ; \\ \mathbb{Q} & \text { if } n=0,1 ; \\ \{0\} & \text { otherwise. }\end{cases}
$$

The Shaneson splitting yields for all $n \in \mathbb{Z}$ an isomorphism

$$
L_{n}^{\langle-\infty\rangle}(\mathbb{Z} W) \cong L_{n}^{\langle-\infty\rangle}\left(\mathbb{Z} W_{0}\right) \oplus L_{n-1}^{\langle-\infty\rangle}\left(\mathbb{Z} W_{0}\right) .
$$

Hence by Theorem 7.12 we find

$$
\mathbb{Z}[1 / 2] \otimes_{\mathbb{Z}} L_{n}^{\langle-\infty\rangle}(\mathbb{Z} W) \cong \begin{cases}\mathbb{Z}[1 / 2]^{12} & \text { if } n=4 k \text { or } n=4 k+1 \text { for } k \in \mathbb{Z} \\ \{0\} & \text { otherwise. }\end{cases}
$$

By (8.1), we get from Theorem 7.16 for all $n \in \mathbb{Z}$

$$
K_{n}\left(C_{r}^{*}(W)\right) \cong \mathbb{Z}^{12}
$$




\section{REFERENCES}

[1] A. Bartels and W. Lück. Induction theorems and isomorphism conjectures for $K$ - and $L$ theory. Forum Math., 19:379-406, 2007.

[2] A. Bartels and W. Lück. The Borel conjecture for hyperbolic and CAT(0)-groups. Ann. of Math. (2), 175:631-689, 2012.

[3] A. C. Bartels. On the domain of the assembly map in algebraic K-theory. Algebr. Geom. Topol., 3:1037-1050 (electronic), 2003.

[4] H. Bass. Algebraic K-theory. W. A. Benjamin, Inc., New York-Amsterdam, 1968.

[5] A. Borel. Stable real cohomology of arithmetic groups. Ann. Sci. École Norm. Sup. (4), 7:235-272 (1975), 1974.

[6] U. Bunke, D. Kasprowski, and C. Winges. Split injectivity of $A$-theoretic assembly maps. Preprint, arXiv:1811.11864 [math.KT], 2018.

[7] S. E. Cappell. Unitary nilpotent groups and Hermitian K-theory. I. Bull. Amer. Math. Soc., 80:1117-1122, 1974.

[8] D. W. Carter. Lower K-theory of finite groups. Comm. Algebra, 8(20):1927-1937, 1980.

[9] R. Charney. An introduction to right-angled Artin groups. Geom. Dedicata, 125:141-158, 2007.

[10] J. F. Davis, Q. Khan, and A. Ranicki. Algebraic K-theory over the infinite dihedral group: an algebraic approach. Algebr. Geom. Topol., 11(4):2391-2436, 2011.

[11] J. F. Davis and W. Lück. Spaces over a category and assembly maps in isomorphism conjectures in $K$ - and L-theory. K-Theory, 15(3):201-252, 1998.

[12] J. F. Davis, F. Quinn, and H. Reich. Algebraic K-theory over the infinite dihedral group: a controlled topology approach. J. Topol., 4(3):505-528, 2011.

[13] M. W. Davis and T. Januszkiewicz. Right-angled Artin groups are commensurable with rightangled Coxeter groups. J. Pure Appl. Algebra, 153(3):229-235, 2000.

[14] A. W. M. Dress. Induction and structure theorems for orthogonal representations of finite groups. Ann. of Math. (2), 102(2):291-325, 1975.

[15] N.-E. Enkelmann, W. Lück, M. Pieper, M. Ullmann, and C. Winges. On the Farrell--Jones conjecture for Waldhausen's A-theory. Geom. Topol., 22(6):3321-3394, 2018.

[16] M. Fuentes Rumí. Equivariant K-theory. Master's thesis, Mathematisches Institut der Universität Bonn, 2018.

[17] G. Gandini and H. Rüping. The Farrell-Jones conjecture for graph products. Algebr. Geom. Topol., 13(6):3651-3660, 2013.

[18] E. Green. Graph products of groups. PhD thesis, The University of Leeds, 1990. Available online at http://etheses.whiterose.ac.uk/236/1/uk_bl_ethos_254954.pdf.

[19] N. Higson and G. Kasparov. E-theory and $K K$-theory for groups which act properly and isometrically on Hilbert space. Invent. Math., 144(1):23-74, 2001.

[20] B. Jahren. Involutions on the rational $K$-theory of group rings of finite groups. In Alpine perspectives on algebraic topology, volume 504 of Contemp. Math., pages 189-202. Amer. Math. Soc., Providence, RI, 2009.

[21] K. H. Kim and F. W. Roush. Homology of certain algebras defined by graphs. J. Pure Appl. Algebra, 17(2):179-186, 1980.

[22] K. Li. On the algebraic $K$-theory for right-angled Coxeter groups. Master's thesis, Mathematisches Institut der Universität Bonn, 2019.

[23] W. Lück. Transformation groups and algebraic K-theory, volume 1408 of Lecture Notes in Mathematics. Springer-Verlag, Berlin, 1989.

[24] W. Lück. Chern characters for proper equivariant homology theories and applications to $K$ and L-theory. J. Reine Angew. Math., 543:193-234, 2002.

[25] W. Lück. $K$-and $L$-theory of the semi-direct product of the discrete 3-dimensional Heisenberg group by $\mathbb{Z} / 4$. Geom. Topol., 9:1639-1676 (electronic), 2005.

[26] W. Lück. Survey on classifying spaces for families of subgroups. In Infinite groups: geometric, combinatorial and dynamical aspects, volume 248 of Progr. Math., pages 269-322. Birkhäuser, Basel, 2005.

[27] W. Lück and H. Reich. The Baum-Connes and the Farrell-Jones conjectures in $K$ - and $L$ theory. In Handbook of K-theory. Vol. 1, 2, pages 703-842. Springer, Berlin, 2005.

[28] W. Lück and W. Steimle. Splitting the relative assembly map, Nil-terms and involutions. Ann. K-Theory, 1(4):339-377, 2016.

[29] J. Milnor. Introduction to algebraic K-theory. Princeton University Press, Princeton, N.J., 1971. Annals of Mathematics Studies, No. 72.

[30] R. Oliver. Whitehead groups of finite groups. Cambridge University Press, Cambridge, 1988.

[31] H. Oyono-Oyono. Baum-Connes Conjecture and extensions. J. Reine Angew. Math., 532:133$149,2001$. 
[32] H. Oyono-Oyono. Baum-Connes conjecture and group actions on trees. K-Theory, 24(2):115134,2001

[33] A. A. Ranicki. Algebraic L-theory and topological manifolds. Cambridge University Press, Cambridge, 1992

[34] R. J. Sánchez-García. Equivariant K-homology for some Coxeter groups. J. Lond. Math. Soc. (2), 75(3):773-790, 2007.

[35] J.-P. Serre. Trees. Springer-Verlag, Berlin, 1980. Translated from the French by J. Stillwell.

[36] R. G. Swan. Induced representations and projective modules. Ann. of Math. (2), 71:552-578, 1960.

[37] T. tom Dieck. Transformation groups and representation theory. Springer-Verlag, Berlin, 1979.

[38] M. Ullmann and C. Winges. On the Farrell-Jones conjecture for algebraic $K$-theory of spaces: the Farrell-Hsiang method. Ann. K-Theory, 4(1):57-138, 2019.

[39] C. T. C. Wall. Norms of units in group rings. Proc. London Math. Soc. (3), 29:593-632, 1974.

[40] C. Wegner. The $K$-theoretic Farrell-Jones conjecture for CAT(0)-groups. Proc. Amer. Math. Soc., 140(3):779-793, 2012.

Mathematisches Institut der Universität Bonn, Endenicher Allee 60, 53115 Bonn, GERMANY

E-mail address: kasprowski@uni-bonn.de

$U R L:$ http://www.math.uni-bonn.de/people/daniel

Mathematisches Institut der Universität Bonn, Endenicher Allee 60, 53115 Bonn, GERMANY

E-mail address: s6keliii@uni-bonn.de

Mathematisches Institut der Universität Bonn, Endenicher Allee 60, 53115 Bonn, Germany

E-mail address: wolfgang.lueck@him.uni-bonn.de

$U R L:$ http://www.him.uni-bonn.de/lueck 